Article

\title{
Epidemiology and Antifungal Susceptibility Patterns of Invasive Fungal Infections (IFIs) in India: A Prospective Observational Study
}

\author{
Yubhisha Dabas ${ }^{1}$, Immaculata Xess ${ }^{1, *}$, Mragnayani Pandey ${ }^{1}$, Jaweed Ahmed ${ }^{1}$, Janya Sachdev ${ }^{1}$ D, Azka Iram ${ }^{1}$, \\ Gagandeep Singh ${ }^{1}{ }^{(D}$, Manoranjan Mahapatra ${ }^{2}$, Rachna Seth ${ }^{3}$, Sameer Bakhshi ${ }^{4}$, Rakesh Kumar ${ }^{5}$, \\ Viveka P. Jyotsna ${ }^{6}$ and Sandeep Mathur ${ }^{7}$
}

check for

updates

Citation: Dabas, Y.; Xess, I.; Pandey, M.; Ahmed, J.; Sachdev, J.; Iram, A.; Singh, G.; Mahapatra, M.; Seth, R.; Bakhshi, S.; et al. Epidemiology and Antifungal Susceptibility Patterns of Invasive Fungal Infections (IFIs) in India: A Prospective Observational Study. J. Fungi 2022, 8, 33. https:// doi.org/10.3390/jof8010033

Academic Editors: Damian J. Krysan and Brian Monk

Received: 30 October 2021

Accepted: 27 December 2021

Published: 30 December 2021

Publisher's Note: MDPI stays neutral with regard to jurisdictional claims in published maps and institutional affiliations.

Copyright: (C) 2021 by the authors. Licensee MDPI, Basel, Switzerland. This article is an open access article distributed under the terms and conditions of the Creative Commons Attribution (CC BY) license (https:// creativecommons.org/licenses/by/ $4.0 /)$.
1 Department of Microbiology, All India Institute of Medical Sciences, New Delhi 110029, India; yubhi.aiims@gmail.com (Y.D.); miggipandey@gmail.com (M.P.); javidamd112@gmail.com (J.A.); drjanyasachdev@gmail.com (J.S.); fineiramkhan@gmail.com (A.I.); drgagandeep@gmail.com (G.S.)

2 Department of Hematology, All India Institute of Medical Sciences, New Delhi 110029, India; mrmahapatra@hotmail.com

3 Department of Paediatrics, All India Institute of Medical Sciences, New Delhi 110029, India; drrachnaseth@yahoo.co.in

4 Department of Medical Oncology, All India Institute of Medical Sciences, New Delhi 110029, India; sambakh@hotmail.com

5 Department of Otorhinolaryngology, All India Institute of Medical Sciences, New Delhi 110029, India; winirk@hotmail.com

6 Department of Endocrinology, All India Institute of Medical Sciences, New Delhi 110029, India; vivekapjyotsna@yahoo.com

7 Department of Pathology, All India Institute of Medical Sciences, New Delhi 110029, India; mathuraiims@yahoo.com

* Correspondence: immaxess@gmail.com; Tel.: +91-98-1826-8181; Fax: +91-11-2659-3208

\begin{abstract}
The epidemiology of invasive fungal infections (IFI) is ever evolving. The aim of the present study was to analyze the clinical, microbiological, susceptibility, and outcome data of IFI in Indian patients to identify determinants of infection and 30-day mortality. Proven and probable/putative IFI (defined according to modified European Organization for Research and Treatment of Cancer/Mycoses Study Group and AspICU criteria) from April 2017 to December 2018 were evaluated in a prospective observational study. All recruited patients were antifungal naïve $(n=3300)$. There were 253 episodes of IFI (7.6\%) with $134(52.9 \%)$ proven and $119(47 \%)$ probable/putative infections. There were four major clusters of infection: invasive candidiasis (IC) $(n=53,20.9 \%)$, cryptococcosis $(n=34$, $13.4 \%$ ), invasive aspergillosis (IA) $(n=103,40.7 \%)$, and mucormycosis ( $n=62,24.5 \%)$. The significant risk factors were high particulate efficiency air (HEPA) room admission, ICU admission, prolonged exposure to corticosteroids, diabetes mellitus, chronic liver disease (CLD), acquired immunodeficiency syndrome (AIDS), coronary arterial disease (CAD), trauma, and multiorgan involvement $(p<0.5$; odds ratio: $>1)$. The all-cause 30 -day mortality was $43.4 \%(n=110)$. It varied by fungal group: $52.8 \%(28 / 53)$ in IC, $58.8 \%(20 / 34)$ in cryptococcosis, $39.8 \%(41 / 103)$ in IA, and $33.9 \%(21 / 62)$ in mucormycosis. HEPA room, ICU admission for IC; HEPA rooms, diabetes mellitus for cryptococcosis; hematological malignancies, chronic kidney disease (CKD), sepsis, galactomannan antigen index value $\geq 1$ for IA and nodules; and ground glass opacities on radiology for mucormycosis were significant predictors of death (odds ratio $>1$ ). High minimum inhibitory concentration (MIC) values for azoles were observed in C. albicans, C. parapsilosis, C. glabrata, A. fumigatus, A. flavus, R. arrhizus, $R$. microsporus, and M. circinelloides. For echinocandin, high MIC values were seen in C. tropicalis, C. guillermondii, C. glabrata, and A. fumigatus. This study highlights the shift in epidemiology and also raises concern of high MICs to azoles among our isolates. It warrants regular surveillance, which can provide the local clinically correlated microbiological data to clinicians and which might aid in guiding patient treatment.
\end{abstract}


Keywords: invasive fungal infections; invasive candidiasis; cryptococcosis; invasive aspergillosis; mucormycosis; risk factor; mortality; antifungal susceptibility

\section{Introduction}

Invasive fungal infections (IFIs) continue to represent a significant problem in immunocompromised individuals and a large proportion of critically ill patients [1]. However, this changing epidemiology with increasing numbers of immunocompetent hosts includes the cases following natural disasters and large iatrogenic inoculation [1,2]. On-going pandemic caused by severe acute respiratory syndrome coronavirus 2 (SARS-CoV-2) has also brought the focus back on superinfections caused by secondary IFIs [3].

Over the past few decades, incidence of IFIs has also been increasing. This is attributed primarily to the overall increase in the number of patients irrespective of severe immunosuppression such as acquired immunodeficiency syndrome (AIDS), hematological malignancies, organ transplantation, etc. or apparent immunocompetent status with diabetes mellitus, chronic obstructive pulmonary disease (COPD), etc. [1,4]. Depending upon the population cohorts, the overall IFI incidence rate varies from 3\% to 20\% [5-11]. Opportunistic pathogens such as Candida sp., Cryptococcus sp., Aspergillus sp., and Mucorales are the most common causative agents of these infections. There are other hyalohyphomycetes such as Fusarium sp. and Scedosporium sp., phaeohyphomycetes (darkly pigmented or dematiaceous fungi), and basidiomycetous yeasts (Trichosporon sp., Malassezia sp.) known to cause these infections in different populations [12]. These fungi affect various tissues throughout the body, with the respiratory system being the most common [13]. Invasive candidiasis is considered the most common IFI; however, there are shifts in epidemiology noted towards non-albicans sp. [4,14]. In hematological diseases, a predominance of invasive aspergillosis (IA) has been reported [1,4]. In critically ill patients, these infections can also present as coinfections, further complicating and delaying the diagnosis [13].

In any economic scenario, the most feasible IFI diagnostic modality-fungal culture and pathological examination-is not conducive, as it does not meet the urgent diagnosis requirement and thereby delays treatment, resulting in a high fatality rate [13]. This is further complicated by the increased occurrence of resistant species owing to a surge in antifungal prophylaxis and emergence of previously rare fungal species displaying inherent resistance to common antifungal agents used $[4,15,16]$. A key determinant for the outcome of IFIs is early initiation of antifungal therapy [17]. There are established guidelines for the four commonest IFIs-invasive candidiasis (IC), Cryptococcosis, invasive aspergillosis (IA), and mucormycosis-from the Infectious Diseases Society of America (IDSA), the European Society for Clinical Microbiology and Infectious Diseases, and the European Confederation of Medical Mycology (ESCMID/ECMM) [18-21]. However, uncertainty lingers about the interpretation of antifungal susceptibility testing (AST) and the significance of minimal inhibitory concentration (MIC) in predicting outcome [17]. Regardless, IFIs are a major cause of morbidity and mortality [4]. Careful consideration of local fungal epidemiology describing clinical characteristics, prognostic factors, use of diagnostic algorithms and antifungal susceptibility patterns can prove useful for overcoming these shortcomings [1].

However, there are a limited number of studies from India, which renders many aspects of IFI poorly understood. This lacuna in data prompted us to conduct this study aimed at analyzing clinical, microbiological, susceptibility, and outcome data of IFIs to support clinicians when deciding on prophylactic or empirical antifungal therapy.

\section{Materials and Methods}

\subsection{Study Design and Data Collection}

This was a prospective observational study to investigate IFI epidemiology from April 2017 to December 2018 conducted at the Department of Microbiology in collaboration with Departments of Hematology, Medical Oncology, Pediatrics, Sleep Disorders and Pulmonary 
Medicine, Otorhinolaryngology, Endocrinology, Medicine and Pathology at a tertiary care hospital, All India Institute of Medical Sciences, New Delhi, India.

Patients clinically suspected of IFI displaying at least one of the following host factors were enrolled in the study: hematologic malignancy; cancer and receiving chemotherapy within the last 3 months before admission, with or without neutropenia; chronic obstructive pulmonary disease (COPD); transplant recipient (hematopoietic/solid organ); chronic granulomatous disease (tuberculosis); other immunocompromised state (inherited immunodeficiency, child C cirrhosis, or HIV, etc.); steroid use-at least $4 \mathrm{mg}$ methylprednisolone (or equivalent) a day for at least 7 days in the 3 weeks before admission or during the course of the ICU stay for at least 5 days or a cumulative dose of at least $250 \mathrm{mg}$ of methylprednisolone (or equivalent) in the past 3 months before enrolment; recipient of any other immunosuppressive treatment (tacrolimus, cyclosporine, methotrexate, cyclophosphamide, etc.); diabetes mellitus with or without ketoacidosis; or microbiological evidence of $A s-$ pergillus infection during the stay in ICU (any positive culture or two positive circulating galactomannan tests) (data not shown). In addition, eligible patients could only be enrolled if they had at least two of the following three features: fever refractory to at least 3 days of appropriate antibiotics or fever relapsing after a period of defervescence of at least $48 \mathrm{~h}$ while still receiving antibiotics; clinical signs and/or symptoms suggestive of invasive mycosis: pleuritic chest pain or physical finding of pleural rub, or one of the following symptoms of lower respiratory tract infection (new sputum secretions, dyspnea, or hemoptysis); or development of new pulmonary infiltrates on chest X-ray. To enhance the homogeneity of the study population, only antifungal-naïve patients were included. The sole exclusion criterion was patients on antifungal prophylaxis or preexisting antifungal treatment. Baseline demographic, clinical characteristics, 30-day all-cause mortality details were recorded. Hospitalization data (general ward/high-efficiency particulate air (HEPA) units/intensive care units (ICU)) were also collected.

Ethics statement: The study was conducted according to the guidelines of the Declaration of Helsinki, and the study was approved by the ethics committee of the institute i.e., All India Institute of Medical Sciences, New Delhi, India (Ref no. IEC/NP25/2014RP-10/2014, OP-3/09.02.2017). The detailed procedure was as per institute guidelines: http://www.aiims.edu/aiims/academic/ethics-committee/forms\%20in\%20pdf/ IEC/Format_of_Institution_Ethics_Committee_15032012.pdf (accessed on 16 January 2017). The consent forms for minor/incapable participants were obtained by their LAR, i.e., legally accepted representatives (example: mother, father, children, or grandparents).

\subsection{Definition of IFI}

Three thousand three hundred consecutive patients who fulfilled European Organization for Research and Treatment of Cancer/Invasive Fungal Infections Cooperative Group and the National Institute of Allergy and Infectious Diseases Mycoses Study Group (EORTC/MSG) 2008 definitions for possible, probable, or proven IFI [22] and AspICU criteria for clinically suspected invasive aspergillosis (IA) in ICUs [23] were enrolled. However, for analysis, only the probable and proven IFIs were included, as per the new EORTC/MSG 2020 definitions [24].

\subsection{Diagnosis of IFI}

Samples were processed following conventional mycological procedures including direct microscopy (visualization of capsule on negative staining, budding yeast cell on grams stain, septate or aseptate hyphae on $\mathrm{KOH}$ mount) and growth on sabouraud dextrose agar and CHROMagar. The isolates were identified by microscopy (slide culture on Tween 80 corn meal agar, septate hyphae on lactophenol cotton blue mount, and aseptate hyphae on calcofluor mount) and morphology on CHROMagar, bird seed agar, malt extract agar, and urea hydrolysis. Galactomannan antigen (GM) assay was performed using Platelia kit (Bio-Rad, Marnes-la-Coquette, France). Serial serum samples (day 0 and day 7) were obtained for all the patients who were clinically suspected of IA for a uniform GM analysis 
as per the physician's recommendation. Capsular antigen of Cryptococcus was detected using latex agglutination test (LAT) of Pastorex ${ }^{\mathrm{TM}}$ Crypto Plus (Bio-Rad, Marnes-la-Coquette, France). Only the isolates difficult to speciate phenotypically were subjected to DNA sequencing, where segments of DNA comprising the ITS region were amplified with primers ITS1 (5'-TCCGTAGGTGAACCTGCGG-3') and ITS4 (5'-TCCTCCGCTTATTGATATGC-3'). Invasive/sterile site samples such as cerebrospinal fluid (CSF), bronchoalveolar lavage (BAL), pleural fluid, and tissue biopsy were collected at the discretion of the attending physician, while considering the debilitated condition of thrombocytopenic patients.

\subsection{Antifungal Susceptibility Patterns}

Antifungal susceptibility testing was performed using the broth microdilution assay according to Clinical Laboratory Standards Institute (CLSI) approved standard M-60 for yeasts [25] and M-38 for molds [26]. Quality control isolates (Candida parapsilosis ATCC 22019, Candida krusei ATCC 6258, and Aspergillus flavus ATCC 204304) were included. All assays were done in duplicates. The antifungal drugs tested were: amphotericin B, flucytosine, itraconazole, fluconazole, voriconazole (Sigma Chemical Corporation, St. Louis, MO, USA); caspofungin, posaconazole (Pfizer Pharmaceuticals, New York, NY, USA); micafungin (Astellas Pharmaceuticals, Tokyo, Japan).

The inoculum suspensions for yeasts were prepared using $0.5 \mathrm{McFarland}$ [25] and for molds conidial suspensions were prepared in RPMI 1640 and adjusted to final concentration of $2.5 \times 10^{4} \mathrm{CFU} / \mathrm{mL}$, as previously described [26]. The assays were incubated at $35^{\circ} \mathrm{C}$ for $24 / 48 \mathrm{~h}$ except for Cryptococcus sp., where the incubation was extended for $72 \mathrm{~h}$.

For Candida sp. the breakpoints followed were according to the M-60 CLSI document [25]. For Cryptococcus sp. and Aspergillus sp., breakpoints used were defined in previous studies from our laboratory $[27,28]$. For mucorales, break points referred by Almyroudis et al. were used for analysis, viz., amphotericin B $\leq 1 \mu \mathrm{g} / \mathrm{mL}$, itraconazole $\leq 0.5 \mu \mathrm{g} / \mathrm{mL}$, and posaconazole $\leq 0.5 \mu \mathrm{g} / \mathrm{mL}[29]$.

\subsection{Statistical Analysis}

Continuous variables are presented as either mean $( \pm S D)$ or median, with interquartile range in case of skewed distribution. They were normally distributed and the Student's t-test was used. The categorical variables are expressed as numbers and percentages of the group from which they were derived. The chi-square test and Fisher's exact test were used to compare categorical variables as appropriate. Socio-demographic clinical characteristics and risk factors were evaluated by univariate and multivariate analysis. These were entered into a logistic regression model for calculation of unpaired and paired odds ratios (ORs). The ORs are given with $95 \%$ confidence intervals (CIs). A cutoff of $p \leq 0.05$, two-tailed, was considered significant for all statistical analysis.

All statistical analysis was conducted using STATA version 9 (StataCorp. 2005. Stata Statistical Software: Release 9. College Station, TX: StataCorp LP) except for antifungal data which was statistically analyzed with Statistical Package for the Social Sciences software (version 16.0; SPSS S.L., Madrid, Spain).

\section{Results}

Three thousand and three hundred patients suspected of IFIs were recruited in the study, of which $253(253 / 3300,7.6 \%)(52 \%, 134 / 253$ proven and $48 \%, 119 / 253$ probable/putative IFIs) presented with $65.6 \%, 166 / 253$ mold (invasive aspergillosis, mucormycosis, and one case of taleromycosis) and $34.4 \%, 87 / 253$ yeast IFIs (invasive candidiasis and cryptococcosis). The case distribution of as proven and probable/putative IFIs is shown in Figure 1. The most common sites of involvement were lung $(42.7 \%, 108 / 253)$ and bloodstream $(20.9 \%, 53 / 253)$ (Table 1). Eighteen episodes of infection were diagnosed by direct microscopy alone. Culture positive infections $(235 / 253,92.9 \%)$ were caused by a wide range of fungal species (Supplementary Figure S1). Species domination was by Candida albicans and Candida parapsilosis $(26.4 \%$ each, $14 / 53)$ in invasive candidiasis cases, 
by Cryptococcus neoformans $(100 \%, 34 / 34)$ in cryptococcosis, by Aspergillus fumigatus $(41.8 \%$, 43/103) and Aspergillus flavus (40.8\%, (42/103) in invasive Aspergillosis, and by Rhizopus arrhizus $(48.4 \%, 30 / 62)$ in mucormycosis (Supplementary Figure S1).

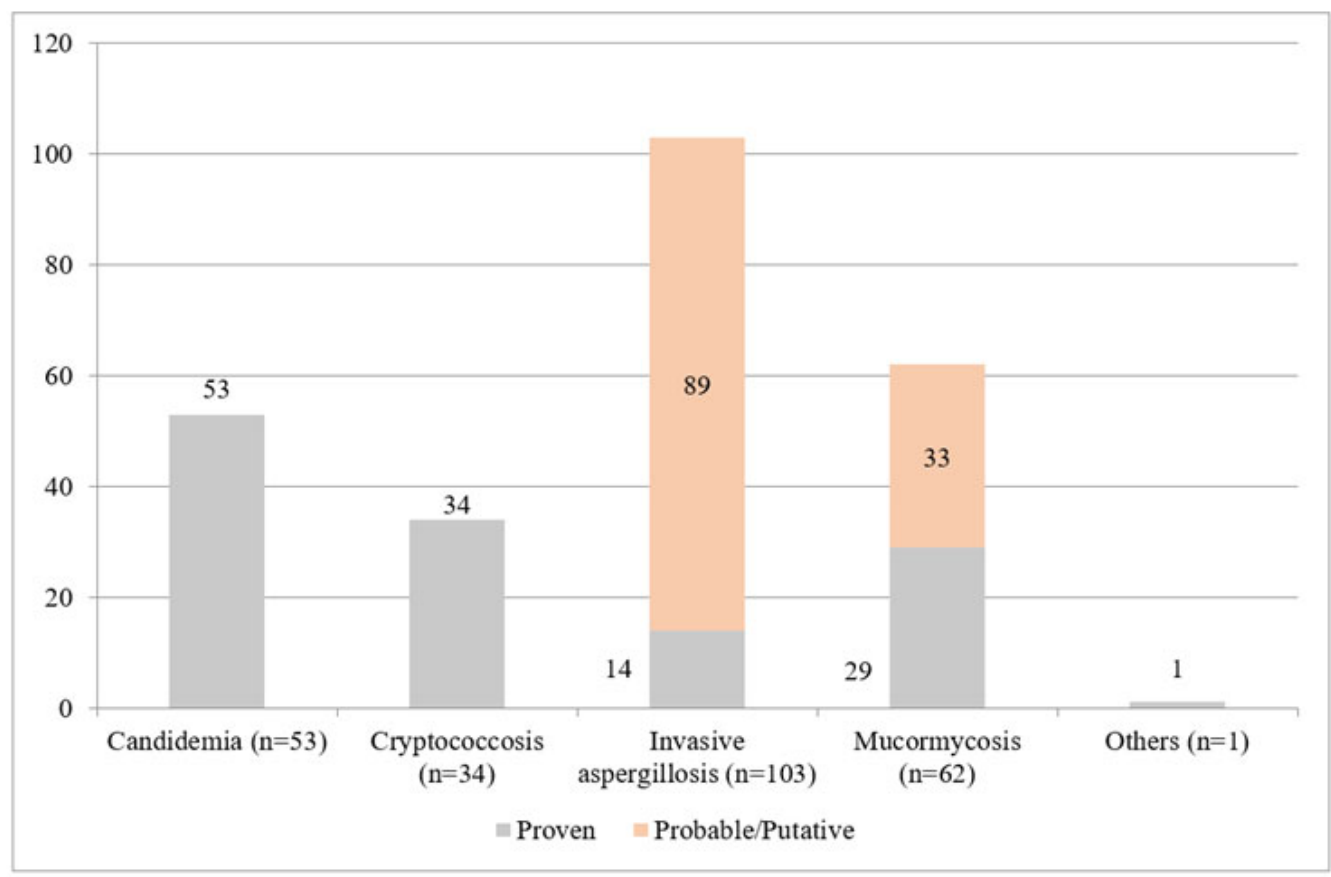

Figure 1. Cases distribution as proven and probable/putative IFI.

Table 1. Site of invasive fungal infections (IFI).

\begin{tabular}{|c|c|c|c|c|c|c|}
\hline Infection Site & $\begin{array}{c}\text { Invasive } \\
\text { Candidiasis } \\
\quad(n=53)\end{array}$ & $\begin{array}{l}\text { Cryptococcosis } \\
\qquad(n=34)\end{array}$ & $\begin{array}{c}\text { Invasive } \\
\text { Aspergillosis } \\
\quad(n=103)\end{array}$ & $\begin{array}{l}\text { Mucormycosis } \\
\quad(n=62)\end{array}$ & Others $(n=1)$ & Total $(n=253)$ \\
\hline Pulmonary (\%) & 0 & $2(5.6)$ & $94(91.3)$ & $12(19.3)$ & 0 & 108 (42.7) \\
\hline Sinus (\%) & 0 & 0 & $6(5.8)$ & $40(64.5)$ & 0 & $46(18.2)$ \\
\hline Blood (\%) & $53(100)$ & 0 & 0 & 0 & 0 & $53(20.9)$ \\
\hline Cerebral (\%) & 0 & $32(94.1)$ & 0 & $2(3.2)$ & 0 & $34(13.4)$ \\
\hline Others (\%) & 0 & 0 & $2(1.9)$ & $2(3.2)$ & $1(100)$ & $5(2)$ \\
\hline Disseminated (\%) & 0 & 0 & $1(0.01)$ & $6(9.7)$ & 0 & $7(2.8)$ \\
\hline
\end{tabular}

The demographic and clinical characteristics describing statistically significant comorbidities and underlying conditions of the patients are listed in Table 2. The majority of subjects were male $(66 \%, 167 / 253)$ and were hospitalized in open general ward $(55.7 \%$, $141 / 253)$. As shown in Figure 1, there were 53\% (134/253) proven IFIs and 47\% (119/253) probable/putative IFIs. High-efficiency particulate air (HEPA) room and ICU hospitalizations were found significantly associated with IFIs (odds ratio, $O R>1$ ). On multivariate analysis, comorbidities such as long term corticosteroids, diabetes mellitus, acquired immunodeficiency syndrome (AIDS), chronic liver disease (CLD), coronary arterial disease $(C A D)$, and trauma were found to be significant predictors of IFI (odds ratio $>1$ ) (Table 2). Individual drugs of choice for definitive treatment were liposomal amphotericin B (30.4\%, $77 / 253)$, followed by voriconazole $(26.9 \%, 68 / 253)$. Overall 30 -day mortality was $43.5 \%$ $(110 / 253)$. 
Table 2. Demographic and clinical characteristics of IFI patients.

\begin{tabular}{|c|c|c|c|c|c|c|}
\hline Variables & $\begin{array}{c}\text { Total } \\
\text { Patients } \\
(n=253)\end{array}$ & $\begin{array}{c}\text { Proven IFI } \\
n=134\end{array}$ & $\begin{array}{c}\text { Probable/ } \\
\text { Putative IFI } \\
n=119\end{array}$ & $p$-Value & $\begin{array}{c}\text { Univariate } \\
\text { OR } \\
(95 \% \mathrm{CI})\end{array}$ & $\begin{array}{c}\text { Multivariate } \\
\text { OR } \\
(95 \% \mathrm{CI})\end{array}$ \\
\hline $\begin{array}{c}\text { Age (years) } \\
\text { Median, IQR (range) }\end{array}$ & $\begin{array}{c}40,33 \\
(0.06-87)\end{array}$ & $\begin{array}{c}35,34 \\
(0.06-83)\end{array}$ & $\begin{array}{l}43,31 \\
(1-87)\end{array}$ & 0.003 & $\begin{array}{c}0.98 \\
(0.97-0.99)\end{array}$ & \\
\hline Males, $n(\%)$ & 167 & $86(51.5)$ & $81(48.5)$ & 0.51 & $\begin{array}{c}0.84 \\
(0.49-1.41)\end{array}$ & \\
\hline $\begin{array}{l}\text { Hospitalization (days) } \\
\text { Median, IQR (range) }\end{array}$ & $\begin{array}{c}19,15 \\
(1-171) \\
\end{array}$ & $\begin{array}{c}21,15 \\
(1-137) \\
\end{array}$ & $\begin{array}{c}17,16 \\
(1-171) \\
\end{array}$ & 0.86 & $0.99(0.98-1)$ & \\
\hline General ward, $n(\%)$ & 141 & $71(50.3)$ & $70(49.6)$ & 0.41 & & \\
\hline $\begin{array}{l}\text { High-efficiency particulate } \\
\text { air-filtered room, } n(\%)\end{array}$ & 56 & $34(60.7)$ & $22(39.3)$ & & $\begin{array}{c}1.52 \\
(0.81-2.86) \\
\end{array}$ & $\begin{array}{c}1.65 \\
(0.8-3.4) \\
\end{array}$ \\
\hline Intensive care unit (ICU), $n(\%)$ & 56 & $29(51.8)$ & $27(48.2)$ & & $\begin{array}{c}1.05 \\
(0.56-1.96) \\
\end{array}$ & $\begin{array}{c}1.2 \\
(0.6-2.4) \\
\end{array}$ \\
\hline $\begin{array}{l}\text { Chronic granulomatous } \\
\quad \text { diseases, } n(\%)\end{array}$ & 50 & $23(46)$ & $27(54)$ & 0.34 & $\begin{array}{c}0.7 \\
(0.37-1.31)\end{array}$ & \\
\hline Long-term corticosteroids, $n(\%)$ & 62 & $40(64.5)$ & $22(35.5)$ & 0.04 & $\begin{array}{c}1.87 \\
(1.03-3.39) \\
\end{array}$ & $\begin{array}{c}2.7 \\
(1.34-5.45) \\
\end{array}$ \\
\hline Diabetes mellitus, $n(\%)$ & 60 & $36(60)$ & $24(40)$ & 0.2 & $1.45(0.8-2.61)$ & $\begin{array}{c}1.3 \\
(0.6-2.8) \\
\end{array}$ \\
\hline $\begin{array}{l}\text { Hematological malignancy, } \\
n(\%)\end{array}$ & 50 & $12(24)$ & $38(76)$ & 0.00 & $\begin{array}{c}0.2 \\
(0.1-0.42)\end{array}$ & \\
\hline Other cancers, $n(\%)$ & 8 & $4(50)$ & $4(50)$ & 1 & $\begin{array}{c}0.88 \\
(0.21-3.61)\end{array}$ & \\
\hline $\begin{array}{l}\text { Acquired immunodeficiency } \\
\text { syndrome, } n(\%)\end{array}$ & 14 & $10(71.4)$ & $4(28.6)$ & 0.16 & $2.31(0.7-7.59)$ & $4.6(1.3-16.6)$ \\
\hline Chronic liver disease, $n(\%)$ & 40 & $33(82.5)$ & $7(17.5)$ & 0.00 & $\begin{array}{c}5.22 \\
(2.21-12.33)\end{array}$ & $6.9(2.8-17.2)$ \\
\hline Pulmonary manifestations, $n(\%)$ & 78 & $18(23.08)$ & $60(76.9)$ & 0.00 & $\begin{array}{c}0.15 \\
(0.08-0.28)\end{array}$ & \\
\hline Chronic kidney disease, $n(\%)$ & 87 & $40(46)$ & $47(54)$ & 0.11 & $\begin{array}{c}0.65 \\
(0.35-1.09) \\
\end{array}$ & \\
\hline Coronary artery disease, $n(\%)$ & 9 & $8(89)$ & $1(11)$ & 0.03 & $\begin{array}{c}7.49 \\
(0.92-60.8) \\
\end{array}$ & $17(2-142)$ \\
\hline Multiorgan involvement, $n(\%)$ & 8 & $8(100)$ & 0 & 0.00 & 1 & \\
\hline Trauma, $n(\%)$ & 15 & $11(73.3)$ & $4(26.7)$ & 0.11 & $2.57(0.79-8.3)$ & $3.9(1-14)$ \\
\hline $\begin{array}{l}\text { Antifungal administration } \\
\text { days median, IQR (range) }\end{array}$ & $\begin{array}{c}14,10 \\
(2-138) \\
\end{array}$ & $14,7(3-87)$ & $21,20(2-138)$ & 0.00 & $\begin{array}{c}0.95 \\
(0.93-0.97)\end{array}$ & \\
\hline Fungal etiology & & & & 0.00 & $\begin{array}{c}0.31 \\
(0.22-0.43) \\
\end{array}$ & \\
\hline Candidemia, $n(\%)$ & 53 & 53 & 0 & & & \\
\hline Cryptococcosis, $n(\%)$ & 34 & 34 & 0 & & & \\
\hline Invasive aspergillosis, $n(\%)$ & 103 & $14(13.6)$ & $89(86.4)$ & & & \\
\hline Mucormycosis, $n(\%)$ & 62 & $33(53.2)$ & $29(46.7)$ & & & \\
\hline Taleromycosis, $n(\%)$ & 1 & 0 & $1(100)$ & & & \\
\hline 30-day outcome, $n(\%)$ & & & & 0.4 & $\begin{array}{c}1.19 \\
(0.72-1.96)\end{array}$ & \\
\hline
\end{tabular}


Table 2. Cont.

\begin{tabular}{|c|c|c|c|c|c|c|}
\hline Variables & $\begin{array}{c}\text { Total } \\
\text { Patients } \\
(n=253)\end{array}$ & $\begin{array}{c}\text { Proven IFI } \\
n=134\end{array}$ & $\begin{array}{c}\text { Probable/ } \\
\text { Putative IFI } \\
n=119\end{array}$ & $p$-Value & $\begin{array}{c}\text { Univariate } \\
\text { OR } \\
(95 \% \text { CI) }\end{array}$ & $\begin{array}{c}\text { Multivariate } \\
\text { OR } \\
(95 \% \text { CI })\end{array}$ \\
\hline Survived, $n(\%)$ & 143 & $73(51)$ & $70(49)$ & & & \\
\hline Expired, $n(\%)$ & 110 & $61(55.4)$ & $49(44.5)$ & & & \\
\hline
\end{tabular}

Note: IFI, invasive fungal infection; $n$, total number of patients; IQR, inter quartile range; OR, odds ratio; CI, confidence interval.

\subsection{Invasive Candidiasis}

All Candida infections were bloodstream. Multiple drugs, single and combinational, were used for treatment with liposomal amphotericin B being used in 35\% (19/53) of cases, followed by a combination of fluconazole with an echinocandin in $20.8 \%(11 / 53)$ of cases (Figure 2). Irrespective of the class of drug, the duration of treatment ranged from 3 to 28 days (median, 11 days; IQR, 7 days). Overall 30-day mortality was $52.8 \%(28 / 53)$. Isolation of Candida tropicalis (OR 4, 95\% CI 0.6-26), Candida parapsilosis (OR 1, 95\% CI 0.2-4.4), and Candida pelliculosa (OR 1, 95\% CI 0.1-19) was associated with poor outcome. On univariate analysis, other variables that significantly predicted mortality were age (OR $1.01,95 \%$ CI $0.98-1.03$ ), sex (OR 1.42, 95\% CI 0.47-4.24), HEPA room hospitalization (OR 2.5, 95\% CI 0.5-12.5), ICU hospitalization (OR 1.7, 95\% CI 0.5-5.8), chronic kidney disease (CKD) (OR 1.9, 95\% CI 0.3-11.4), pulmonary manifestations (OR 1.14, 95\% CI 0.26-4.8), and multiorgan involvement (OR 3.13, 95\% CI 0.57-17.2).

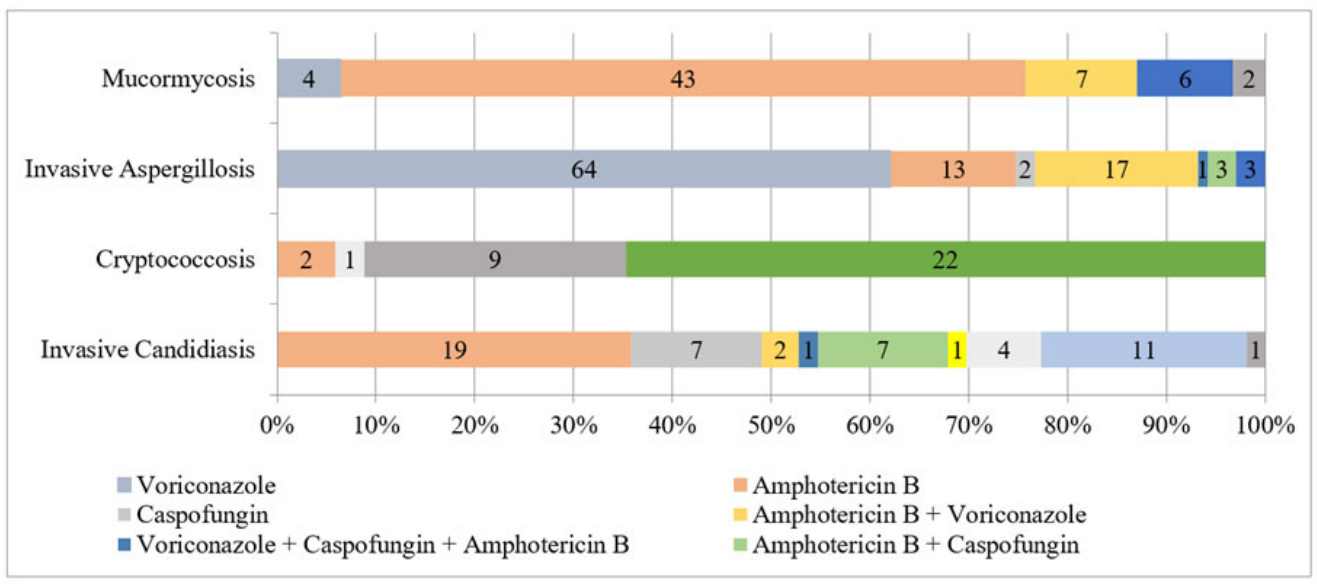

Figure 2. Antifungal treatment in IFI.

\subsection{Cryptococcosis}

Other than two pulmonary cases $(5.6 \%, 2 / 34)$, all had cerebral presentation $(94.4 \%$, $32 / 34)$. A total of $24(70.6 \%, 24 / 34)$ were culture positive with Cryptococcus neoformans, whereas $10(29.4 \%)$ only showed budding round yeast cell with halo on india ink staining. Latex agglutination testing for the capsular antigen was carried out for all patients (data not shown). Clinical characteristics noted are shown in Figure 3. Flucytosine with Lamphotericin B was given in 64.7\% (22/34) of cases, and fluconazole with L-amphotericin B was treatment of choice in $24.5 \%$ (9/34) of cases. Overall 30-day mortality was 58.8\% (20/34). Patients were mostly admitted in open general wards $25 / 34(73.5 \%)$, with only nine in HEPA room hospitalizations (26.5\%), and admission in the latter was associated with poor outcome $p=0.19$ (OR 3.23, 95\% CI 0.55-18.7). On univariate analysis, significant predictors of mortality were hospitalization duration (OR 1.01, 95\% CI 0.85-1.2), chronic granulomatous diseases (CGD) (OR 1.33, 95\% CI 0.33-5.26), diabetes mellitus (OR 2.29, 95\% CI 0.21-24.6), AIDS (OR 1.97, 95\% CI 0.4-9.5), and CKD (OR 1, 95\% CI 0.25-3.91). 




Figure 3. Clinical characteristics of Cryptococcosis.

\subsection{Invasive Aspergillosis}

The cases were mainly of invasive pulmonary aspergillosis $(91.3 \%, 94 / 103)$. The treatment of choice was voriconazole in $61.5 \%(64 / 103)$ cases, followed by a combination of L-amphotericin B and voriconazole in 16.5\% (17/103) cases. All were direct microscopy and culture positive. Single isolates from repeat isolations were included for respiratory non-invasive samples (data not shown). Galactomannan antigen testing was performed for all patients (data not shown). Radiological findings are shown in Figure 4. Overall 30-day mortality was 39.8\% (41/103). Isolation of Aspergillus fumigatus (OR 1.9, 95\% CI 0.8-4.6), Aspergillus nidulans (OR 1, 95\% CI 0.08-12), and Aspergillus niger (OR 1.2, 95\% CI 0.25-5.7) was associated with poor outcome. Administration of L-amphotericin B (OR 1.04, 95\% CI 0.3-3.55) and a combination of the former with posaconazole (OR 3.33, 95\% CI 0.28-38.7) was associated with poor outcome. Radiological picture suggestive of lung collapse (OR 1.5, 95\% CI 0.05-40.6) and appearance of nodules and ground glass opacities (OR 1.5, 95\% CI $0.22-9.96)$ also predicted mortality. On univariate analysis, other variables that significantly predicted mortality were age (OR 1.03, 95\% CI 1.01-1.05), sex (OR 1.1, 95\% CI 0.47-2.56), ICU hospitalization (OR 4.27, 95\% CI 1.73-10.53), hematological malignancy (OR 2.48, 95\% CI 1.07-5.73), CKD (OR 3.67, 95\% CI 1.6-8.5), prolonged corticosteroids (OR 1.56, $95 \%$ CI 0.7-3.48), mechanical ventilation (OR 2.77, 95\% CI 1.21-6.36), sepsis (OR 3.67, $95 \%$ CI 1.15-11.72), and high galactomannan antigen index value of $\geq 1$ (OR 1.6, 95\% CI $0.72-3.56)$.

\subsection{Mucormycosis}

Fifty percent (31/62) of cases were rhino-orbital with sinus involvement. Overall site and tissue involvement is shown in Figure 5. All were direct microscopy positive with $87 \%$ $(54 / 62)$ of culture growth. Surgical debridement was performed in $71 \%(44 / 62)$ of cases, with L-amphotericin B $(43 / 62,69 \%)$ as the most common antifungal used. Overall 30-day mortality was $33.9 \%$ (21/62). Rhizopus microsporus (OR 1.94, 95\% CI 0.32-11.75) and Mucor isolations (OR 3.33, 95\% CI 0.2-54.5) were associated with poor outcome. On radiology, appearance of consolidation (OR 1.2, 95\% CI 0.08-16.4), nodules/ground glass opacities (OR 8.4, 95\% CI 1.27-55.4), and sinus thickening (OR 2.05, 95\% CI 0.45-9.3) were associated with poor outcome. On univariate analysis, other significant predictors of mortality were age (OR 3.33, 95\% CI 0.2-54.5), hematological malignancy (OR 3.33, 95\% CI 0.2-54.5), CKD (OR 3.33, 95\% CI 0.2-54.5), symptoms duration (OR 3.33, 95\% CI 0.2-54.5), pulmonary 
mucormycosis (OR 3.33, 95\% CI 0.2-54.5), and ketoacidosis (OR 3.33, 95\% CI 0.2-54.5). Multivariate analysis of mortality predictors for all IFIs is listed in Table 3.

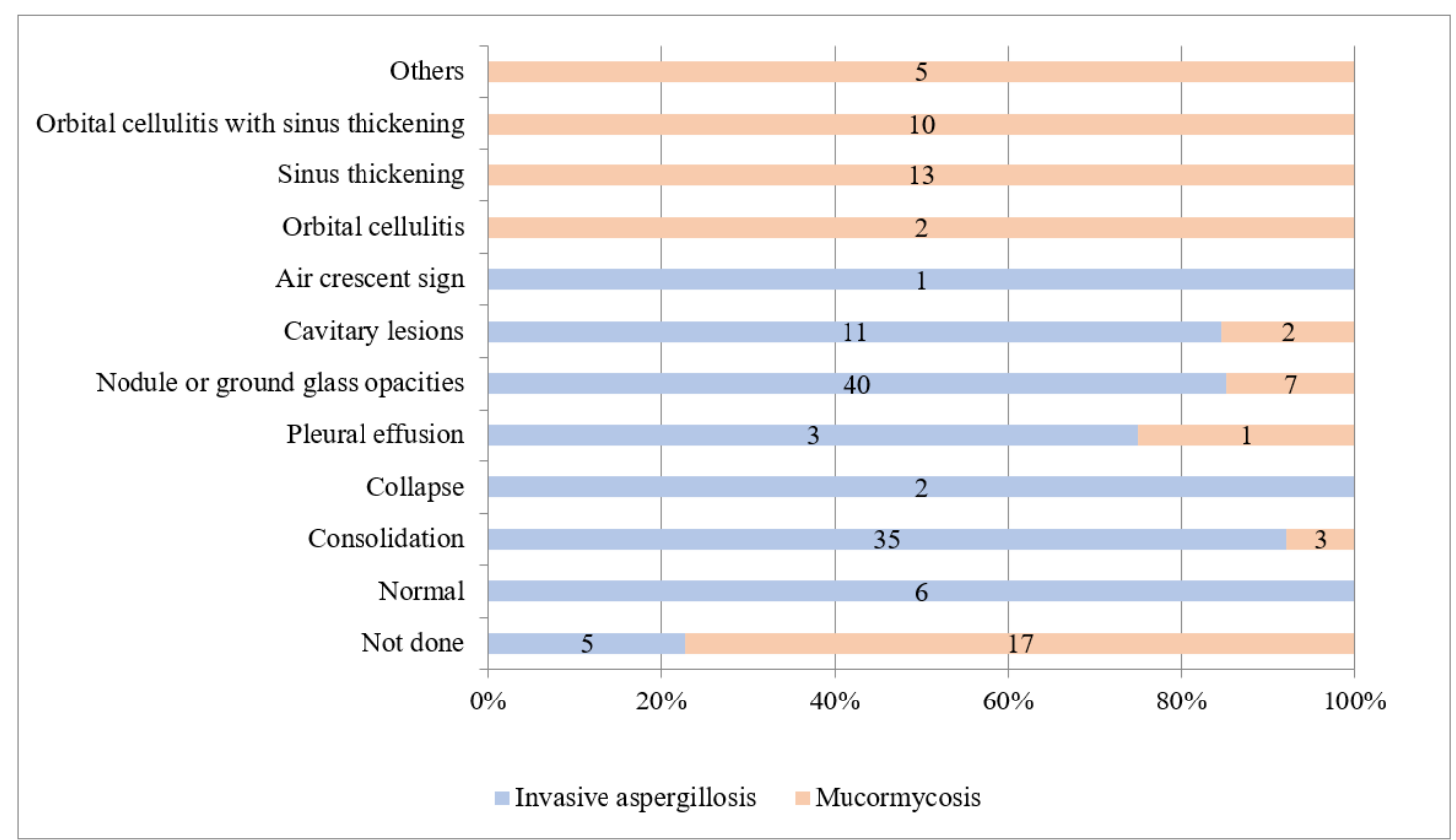

Figure 4. Radiological findings in invasive aspergillosis and mucormycosis cases.



Figure 5. Site and tissue involvement in mucormycosis.

\subsection{Antifungal Susceptibility Testing}

All experiments were performed in duplicates, and the MIC values of quality control strains fell within the established ranges published by CLSI methodologies. Table 4 summarizes the in vitro susceptibility value ranges, geometric mean, mode, $\mathrm{MIC}_{50}$, and $\mathrm{MIC}_{90}$ values of all the isolates to the antifungals tested. Irrespective of genera, all isolates were susceptible to amphotericin B. Based on breakpoints for different fungi, high MIC values (intermediate and resistant combined) were recorded for fluconazole in 3 C. albicans, 4 C. parapsilosis, and 1 C. guillermondii; for voriconazole in 3 C. albicans, 3 C. parapsilosis, and 5 C. glabrata; for itraconazole in 4 C. albicans, 4 C. parapsilosis, 3 C. glabrata, 1 A. flavus, 1 A. fumigatus, 1 A. niger, 12 R. arrhizus, 3 R. microsporus, and 1 Mucor circinelloides; for posaconazole in $15 \mathrm{R}$. arrhizus, and $7 \mathrm{R}$. microsporus; for caspofungin in $1 \mathrm{C}$. albicans, $2 \mathrm{C}$. tropicalis, 4 C. glabrata, and 2 A. fumigatus; and for micafungin in 3 C. tropicalis, 2 C. guillermondii, and 4 C. glabrate (Table 5). 
Table 3. Multivariate analysis showing the interdependent variables as significant predictors of mortality in different IFI.

\begin{tabular}{|c|c|c|c|c|}
\hline Variables & $\begin{array}{c}\text { Invasive } \\
\text { Candidiasis } \\
\text { OR }(95 \% \mathrm{CI})\end{array}$ & $\begin{array}{l}\text { Cryptococcosis } \\
\text { OR }(95 \% \text { CI })\end{array}$ & $\begin{array}{c}\text { Invasive } \\
\text { Aspergillosis } \\
\text { OR (95\% CI) }\end{array}$ & $\begin{array}{l}\text { Mucormycosis } \\
\text { OR }(95 \% \text { CI })\end{array}$ \\
\hline Age & $1.07(1.01-1.14)$ & & $1.07(1.03-1.11)$ & $1.01(0.96-1.06)$ \\
\hline Sex & $3.27(0.52-20.46)$ & & $1.39(0.29-6.6)$ & \\
\hline Hospitalization duration & & $1.04(0.85-1.27)$ & & \\
\hline $\begin{array}{l}\text { High-efficiency particulate } \\
\text { air-filtered room }\end{array}$ & $31.14(1.72-560)$ & $5.3(0.75-37.38)$ & & \\
\hline Intensive care unit (ICU) & $10.49(0.99-110.3)$ & & $3.15(0.84-11.75)$ & \\
\hline $\begin{array}{c}\text { Chronic granulomatous } \\
\text { diseases }\end{array}$ & & $1.08(0.09-12.55)$ & & \\
\hline Long-term corticosteroids & & & $2.63(0.6-11.9)$ & \\
\hline Diabetes mellitus & & $3.77(0.19-74.48)$ & & \\
\hline Hematological malignancy & & & $15(2.9-77.9)$ & \\
\hline $\begin{array}{l}\text { Acquired immunodeficiency } \\
\text { syndrome }\end{array}$ & & $3.4(0.23-48.94)$ & & \\
\hline Pulmonary manifestations & $2.95(0.33-26.06)$ & & & $1.35(0.4-4.6)$ \\
\hline Chronic kidney disease & & $1.07(0.15-7.42)$ & $3.9(1.1-13.7)$ & \\
\hline Multiorgan involvement & $9.5(0.94-95.7)$ & & & \\
\hline Mechanical ventilation & & & $2.99(0.76-11.63)$ & \\
\hline Sepsis & & & $5.8(0.79-42.3)$ & \\
\hline Symptom duration & & & & $1.08(0.83-1.4)$ \\
\hline Ketoacidosis & & & & $1.13(0.12-10.68)$ \\
\hline \multicolumn{5}{|l|}{ Radiological finding } \\
\hline Lung collapse & & & $1.5(0.05-40.6)$ & \\
\hline $\begin{array}{c}\text { Nodules/ground glass } \\
\text { opacities }\end{array}$ & & & $1.5(0.22-9.96)$ & $100.5(1.44-7006)$ \\
\hline Consolidation & & & & 9.69 (0.09-997) \\
\hline Sinus thickening & & & & $1.02(0.13-7.57)$ \\
\hline $\begin{array}{l}\text { Galactomannan antigen } \\
\text { index } \geq 1\end{array}$ & & & $2.72(0.76-9.65)$ & \\
\hline \multicolumn{5}{|l|}{ Species isolation } \\
\hline & $\begin{array}{l}\text { Candida tropicalis: } 12.9 \\
(1.11-150)\end{array}$ & & & $\begin{array}{c}\text { Rhizopus } \\
\text { microsporus: } 1.2 \\
(0.09-15.5)\end{array}$ \\
\hline & $\begin{array}{l}\text { C. parapsilosis: } 1.2 \\
(0.18-7.9)\end{array}$ & & & $\begin{array}{c}\text { Mucor } \\
\text { circinelloides: } 2.06 \\
(0.01-328.9)\end{array}$ \\
\hline & $\begin{array}{l}\text { C. pelliculosa: } 1.2 \\
(0.03-43.5)\end{array}$ & & & \\
\hline
\end{tabular}

Note: IFI, invasive fungal infections; OR, odds ratio; CI, confidence interval. 
Table 4. MIC range with geometric mean, mode, $\mathrm{MIC}_{50}$, and $\mathrm{MIC}_{90}$ values for the different fungal species from IFI cases by CLSI methodology.

\begin{tabular}{|c|c|c|c|c|c|c|c|c|c|c|c|c|c|c|c|c|c|}
\hline \multirow[b]{2}{*}{ Fungal Isolate } & \multirow[b]{2}{*}{$N$} & \multicolumn{2}{|c|}{ Amphotericin B } & \multicolumn{2}{|c|}{ Fluconazole } & \multicolumn{2}{|c|}{ Voriconazole } & \multicolumn{2}{|c|}{ Itraconazole } & \multicolumn{2}{|c|}{ Posaconazole } & \multicolumn{2}{|c|}{ Caspofungin } & \multicolumn{2}{|c|}{ Micafungin } & \multicolumn{2}{|c|}{ Flucytosine } \\
\hline & & $\begin{array}{l}\text { MIC }_{50} / \text { MIC }_{90} \\
\text { MIC Range }\end{array}$ & $\begin{array}{l}\text { Mode, } \\
\text { GM }\end{array}$ & $\begin{array}{l}\text { MIC }_{50} / \mathrm{MIC}_{90} ; \\
\text { MIC Range }\end{array}$ & $\begin{array}{l}\text { Mode, } \\
\text { GM }\end{array}$ & $\begin{array}{l}\text { MIC }_{50} / \text { MIC }_{90} ; \\
\text { MIC Range }\end{array}$ & $\begin{array}{l}\text { Mode, } \\
\text { GM }\end{array}$ & $\begin{array}{l}\text { MIC }_{50} / \mathrm{MIC}_{90} \\
\text { MIC Range }\end{array}$ & $\begin{array}{l}\text { Mode, } \\
\text { GM }\end{array}$ & $\begin{array}{l}\text { MIC }_{50} / \text { MIC }_{90} ; \\
\text { MIC Range }\end{array}$ & $\begin{array}{c}\text { Mode, } \\
\text { GM }\end{array}$ & $\begin{array}{l}\text { MIC }_{50} / \mathrm{MIC}_{90} ; \\
\text { MIC Range }\end{array}$ & $\begin{array}{l}\text { Mode, } \\
\text { GM }\end{array}$ & $\begin{array}{l}\text { MIC }_{50} / \text { MIC }_{90} ; \\
\text { MIC Range }\end{array}$ & $\begin{array}{l}\text { Mode, } \\
\text { GM }\end{array}$ & $\begin{array}{l}\text { MIC }_{50} / \mathrm{MIC}_{90} \\
\text { MIC Range }\end{array}$ & $\begin{array}{l}\text { Mode, } \\
\text { GM }\end{array}$ \\
\hline Candida sp. & 53 & $\begin{array}{c}0.25 / 0.5 ; \\
0.03-1\end{array}$ & $\begin{array}{c}0.5 \\
0.217 \\
\end{array}$ & $\begin{array}{c}0.5 / 8 \\
0.125-64 \\
\end{array}$ & $\begin{array}{l}0.25, \\
0.812 \\
\end{array}$ & $\begin{array}{c}0.03 / 0.25 \\
0.03-0.5\end{array}$ & $\begin{array}{l}0.03, \\
0.063 \\
\end{array}$ & $\begin{array}{c}0.06 / 0.5 ; \\
0.03-1\end{array}$ & $\begin{array}{l}0.03 \\
0.081 \\
\end{array}$ & $\begin{array}{c}0.03 / 0.5 ; \\
0.03-1\end{array}$ & $\begin{array}{l}0.03 \\
0.054 \\
\end{array}$ & $\begin{array}{c}0.125 / 0.5 ; \\
0.015-1\end{array}$ & $\begin{array}{l}0.015 \\
0.097 \\
\end{array}$ & $\begin{array}{c}0.015 / 0.5 ; \\
0.015-2\end{array}$ & $\begin{array}{l}0.015 \\
0.047 \\
\end{array}$ & & \\
\hline $\begin{array}{l}\text { Candida } \\
\text { albicans }\end{array}$ & 14 & $0.06-0.5$ & $\begin{array}{l}0.5, \\
0.26\end{array}$ & $0.125-64$ & $\begin{array}{l}0.25 \\
0.82\end{array}$ & $0.03-0.5$ & $\begin{array}{l}0.03, \\
0.07\end{array}$ & $0.03-1$ & $\begin{array}{l}0.03, \\
0.11\end{array}$ & $0.03-0.5$ & $\begin{array}{l}0.03 \\
0.05\end{array}$ & $0.015-0.5$ & $\begin{array}{l}0.25, \\
0.08\end{array}$ & $0.015-1$ & $\begin{array}{l}0.015 \\
0.035\end{array}$ & & \\
\hline C. tropicalis & 10 & $0.03-0.5$ & $\begin{array}{l}0.03, \\
0.107\end{array}$ & $0.25-2$ & $\begin{array}{c}0.5, \\
0.466\end{array}$ & $0.03-0.125$ & $\begin{array}{l}0.03, \\
0.039 \\
\end{array}$ & $0.03-0.125$ & $\begin{array}{l}0.03, \\
0.045 \\
\end{array}$ & $0.03-0.5$ & $\begin{array}{l}0.03 \\
0.056 \\
\end{array}$ & $0.015-0.5$ & $\begin{array}{l}0.125 \\
0.107\end{array}$ & $0.015-1$ & $\begin{array}{l}0.015 \\
0.065\end{array}$ & & \\
\hline C. parapsilosis & 14 & $0.125-1$ & $\begin{array}{c}0.5, \\
0.304\end{array}$ & $0.125-64$ & $\begin{array}{c}0.5, \\
1.034 \\
\end{array}$ & $0.03-0.5$ & $\begin{array}{l}0.03, \\
0.063 \\
\end{array}$ & $0.03-1$ & $\begin{array}{l}0.03, \\
0.081 \\
\end{array}$ & $0.03-1$ & $\begin{array}{l}0.03 \\
0.054 \\
\end{array}$ & $0.015-1$ & $\begin{array}{l}0.25 \\
0.105 \\
\end{array}$ & $0.015-0.5$ & $\begin{array}{l}0.015 \\
0.042\end{array}$ & & \\
\hline $\begin{array}{c}C . \\
\text { guillermondii }\end{array}$ & 2 & $0.03-0.25$ & & $0.5-32$ & & $0.03-0.06$ & & $0.03-0.25$ & & $0.125-0.5$ & & $0.125-1$ & & $0.5-2$ & & & \\
\hline C. pelliculosa & 2 & $0.125-0.25$ & & $0.25-2$ & & 0.03 & & 0.03 & & 0.03 & & $0.015-0.06$ & & $0.015-0.5$ & & & \\
\hline C. auris & 2 & 0.125 & & $0.125-0.25$ & & 0.03 & & 0.03 & & 0.03 & & $0.03-0.06$ & & 0.015 & & & \\
\hline C. glabrata & 8 & $0.125-0.5$ & $\begin{array}{c}0.5, \\
0.272 \\
\end{array}$ & $0.25-8$ & 1,1 & $0.03-0.5$ & $\begin{array}{l}0.25, \\
0.123 \\
\end{array}$ & $0.06-0.5$ & $\begin{array}{l}0.06, \\
0.122 \\
\end{array}$ & $0.03-2$ & $\begin{array}{l}0.03, \\
0.06\end{array}$ & $0.015-1$ & $\begin{array}{c}0.125 \\
0.16\end{array}$ & $0.015-0.5$ & $\begin{array}{l}0.015 \\
0.072\end{array}$ & & \\
\hline $\begin{array}{l}\text { Lodderomyces } \\
\text { longisporus }\end{array}$ & 1 & 0.125 & & 0.5 & & 0.03 & & 0.03 & & 0.03 & & 0.015 & & 0.015 & & & \\
\hline $\begin{array}{l}\text { Cryptococcus } \\
\text { neoformans }\end{array}$ & 24 & $\begin{array}{l}0.25 / 1 ; \\
0.03-1\end{array}$ & $\begin{array}{l}0.25 \\
0.342\end{array}$ & $2 / 4 ; 0.5-8$ & $2,2.181$ & $\begin{array}{l}0.03 / 0.06 ; \\
0.03-0.06\end{array}$ & $\begin{array}{l}0.03, \\
0.033\end{array}$ & & & & & & & & & $\begin{array}{c}2 / 2 \\
0.25-4\end{array}$ & $2,1.414$ \\
\hline Aspergillus sp. & 103 & $1 / 2 ; 0.03-4$ & $1,0.831$ & & & $\begin{array}{l}0.5 / 1 \\
0.03-2\end{array}$ & $\begin{array}{r}0.5 \\
0.285\end{array}$ & $\begin{array}{l}0.5 / 1 \\
0.03-2\end{array}$ & $\begin{array}{r}0.5 \\
0.259\end{array}$ & $\begin{array}{l}0.06 / 0.25 \\
0.03-0.25\end{array}$ & $\begin{array}{c}0.03 \\
0.081\end{array}$ & $\begin{array}{c}0.06 / 0.125 \\
0.015-1\end{array}$ & $\begin{array}{l}0.015 \\
0.046\end{array}$ & $\begin{array}{c}0.015 / \\
0.015 \\
0.015-0.03\end{array}$ & $\begin{array}{l}0.015 \\
0.016\end{array}$ & & \\
\hline $\begin{array}{l}\text { Aspergillus } \\
\text { flavus }\end{array}$ & 42 & $2 / 4 ; 0.06-4$ & $2,1.559$ & & & $\begin{array}{l}0.5 / 1 ; \\
0.03-1\end{array}$ & $\begin{array}{c}0.5 \\
0.363 \\
\end{array}$ & $\begin{array}{l}0.25 / 1 ; \\
0.03-2\end{array}$ & $\begin{array}{r}0.5, \\
0.255 \\
\end{array}$ & $\begin{array}{c}0.06 / 0.25 \\
0.03-0.5\end{array}$ & $\begin{array}{l}0.03 \\
0.085 \\
\end{array}$ & $\begin{array}{l}0.06 / 0.125 ; \\
0.015-0.25\end{array}$ & $\begin{array}{r}0.06 \\
0.045 \\
\end{array}$ & $\begin{array}{c}0.015 / 0.015 \\
0.015-0.03\end{array}$ & $\begin{array}{l}0.015 \\
0.016 \\
\end{array}$ & & \\
\hline A. fumigatus & 43 & $\begin{array}{c}0.5 / 2 \\
0.03-2\end{array}$ & $1,0.539$ & & & $\begin{array}{l}0.25 / 1 ; \\
0.03-1\end{array}$ & $\begin{array}{l}0.5, \\
0.24\end{array}$ & $\begin{array}{l}0.25 / 1 ; \\
0.03-2\end{array}$ & $\begin{array}{c}0.5, \\
0.251\end{array}$ & $\begin{array}{c}0.06 / 0.25 \\
0.03-0.5\end{array}$ & $\begin{array}{l}0.03 \\
0.067\end{array}$ & $\begin{array}{c}0.06 / 0.125 \\
0.015-1\end{array}$ & $\begin{array}{l}0.015 \\
0.049\end{array}$ & $\begin{array}{c}0.015 / 0.015 \\
0.015-0.03\end{array}$ & $\begin{array}{l}0.015 \\
0.015\end{array}$ & & \\
\hline A. terreus & 7 & $0.5-1$ & $1,0.905$ & & & $0.06-1$ & $\begin{array}{c}0.5 \\
0.272\end{array}$ & $0.125-0.5$ & $\begin{array}{l}0.5, \\
0.25\end{array}$ & $0.03-0.5$ & $\begin{array}{l}0.125 \\
0.124\end{array}$ & $0.03-0.25$ & $\begin{array}{l}0.06 \\
0.044\end{array}$ & $0.015-0.03$ & $\begin{array}{l}0.015 \\
0.016\end{array}$ & & \\
\hline A. nidulans & 3 & $0.06-1$ & $1,0.391$ & & & $0.06-0.5$ &,- 0.155 & $0.03-0.5$ & $\begin{array}{c}0.5 \\
0.195\end{array}$ & $0.03-0.125$ & $\begin{array}{l}0.125 \\
0.077\end{array}$ & $0.03-0.125$ &,- 0.06 & $0.015-0.03$ & $\begin{array}{l}0.015 \\
0.018\end{array}$ & & \\
\hline A. niger & 8 & $0.06-2$ & $\begin{array}{l}0.25 \\
0.383 \\
\end{array}$ & & & $0.06-2$ & $\begin{array}{l}0.06, \\
0.268\end{array}$ & $0.06-2$ & $\begin{array}{c}0.5, \\
0.383 \\
\end{array}$ & $0.03-0.5$ & $\begin{array}{l}0.25 \\
0.134\end{array}$ & $0.015-0.25$ & $\begin{array}{l}0.015 \\
0.030\end{array}$ & 0.015 & $\begin{array}{l}0.015 \\
0.015\end{array}$ & & \\
\hline Mucorales & 54 & $\begin{array}{c}0.125 / 0.5 ; \\
0.06-1\end{array}$ & $\begin{array}{l}0.06, \\
0.138\end{array}$ & & & & & $\begin{array}{l}0.5 / 1 ; \\
0.06-1\end{array}$ & $\begin{array}{c}0.5 \\
0.450\end{array}$ & $\begin{array}{l}0.25 / 1 \\
0.03-2\end{array}$ & $\begin{array}{r}0.25 \\
0.361\end{array}$ & & & & & & \\
\hline
\end{tabular}


Table 4. Cont.

\begin{tabular}{|c|c|c|c|c|c|c|c|c|c|c|c|c|c|c|c|c|c|}
\hline \multirow[b]{2}{*}{ Fungal Isolate } & \multirow[b]{2}{*}{$N$} & \multicolumn{2}{|c|}{ Amphotericin B } & \multicolumn{2}{|c|}{ Fluconazole } & \multicolumn{2}{|c|}{ Voriconazole } & \multicolumn{2}{|c|}{ Itraconazole } & \multicolumn{2}{|c|}{ Posaconazole } & \multicolumn{2}{|c|}{ Caspofungin } & \multicolumn{2}{|c|}{ Micafungin } & \multicolumn{2}{|c|}{ Flucytosine } \\
\hline & & $\begin{array}{l}\mathrm{MIC}_{50} / \mathrm{MIC}_{90} ; \\
\text { MIC Range }\end{array}$ & $\begin{array}{l}\text { Mode, } \\
\text { GM }\end{array}$ & $\begin{array}{l}\text { MIC }_{50} / \mathrm{MIC}_{90} ; \\
\text { MIC Range }\end{array}$ & $\begin{array}{c}\text { Mode, } \\
\text { GM }\end{array}$ & $\begin{array}{l}\text { MIC }_{50} / \mathrm{MIC}_{90} \\
\text { MIC Range }\end{array}$ & $\begin{array}{l}\text { Mode, } \\
\text { GM }\end{array}$ & $\begin{array}{l}\mathrm{MIC}_{50} / \mathrm{MIC}_{90} \\
\text { MIC Range }\end{array}$ & $\begin{array}{l}\text { Mode, } \\
\text { GM }\end{array}$ & $\begin{array}{l}\text { MIC }_{50} / \mathrm{MIC}_{90} ; \\
\text { MIC Range }\end{array}$ & $\begin{array}{l}\text { Mode, } \\
\text { GM }\end{array}$ & $\begin{array}{l}\text { MIC }_{50} / \mathrm{MIC}_{90} ; \\
\text { MIC Range }\end{array}$ & $\begin{array}{l}\text { Mode, } \\
\text { GM }\end{array}$ & $\begin{array}{l}\text { MIC }_{50} / \mathrm{MIC}_{90} ; \\
\text { MIC Range }\end{array}$ & $\begin{array}{l}\text { Mode, } \\
\text { GM }\end{array}$ & $\begin{array}{l}\mathrm{MIC}_{50} / \mathrm{MIC}_{90} ; \\
\text { MIC Range }\end{array}$ & $\begin{array}{c}\text { Mode, } \\
\text { GM }\end{array}$ \\
\hline $\begin{array}{l}\text { Rhizopus } \\
\text { arrhizus }\end{array}$ & 30 & $\begin{array}{c}0.125 / 0.25 \\
0.06-1\end{array}$ & $\begin{array}{l}0.125 \\
0.135\end{array}$ & & & & & $\begin{array}{l}0.5 / 1 \\
0.06-1\end{array}$ & $1,0.499$ & $\begin{array}{l}0.25 / 2 ; \\
0.03-2\end{array}$ & $\begin{array}{l}0.25 \\
0.475\end{array}$ & & & & & & \\
\hline R. microsporus & 13 & $0.06-0.5$ & $\begin{array}{l}0.06, \\
0.115 \\
\end{array}$ & & & & & $0.25-1$ & $0.5,0.5$ & $0.03-1$ & $1,0.360$ & & & & & & \\
\hline R.pusillus & 1 & 0.125 & & & & & & 0.125 & & 0.25 & & & & & & & \\
\hline $\begin{array}{l}\text { Lichtheimia } \\
\text { corymbifera }\end{array}$ & 4 & 0.25 & $\begin{array}{l}0.25, \\
0.25\end{array}$ & & & & & $0.125-0.25$ & $\begin{array}{l}0.25, \\
0.21 \\
\end{array}$ & 0.125 & $\begin{array}{l}0.125, \\
0.125 \\
\end{array}$ & & & & & & \\
\hline $\begin{array}{c}\text { Apophysomyces } \\
\text { variabilis }\end{array}$ & 1 & 0.06 & & & & & & 0.25 & & 0.03 & & & & & & & \\
\hline $\begin{array}{c}\text { Mucor } \\
\text { circinelloides }\end{array}$ & 3 & 0.25 & $\begin{array}{l}0.25, \\
0.25\end{array}$ & & & & & $0.5-1$ & $\begin{array}{c}0.5 \\
0.629\end{array}$ & $0.25-0.5$ & $\begin{array}{l}0.25 \\
0.314\end{array}$ & & & & & & \\
\hline $\begin{array}{l}\text { Conidiobolus } \\
\text { coronatus }\end{array}$ & 1 & 0.06 & & & & & & 0.25 & & 0.25 & & & & & & & \\
\hline $\begin{array}{c}\text { Taleromyces } \\
\text { marneffi }\end{array}$ & 1 & 0.125 & & & & 0.06 & & 0.125 & & 0.03 & & 0.015 & & 0.015 & & & \\
\hline
\end{tabular}

Note: $N$, total number of isolates; MIC, minimum inhibitory concentration; GM, geometric mean, -, not calculated. 
Table 5. MIC interpretation for various fungi from IFI cases.

\begin{tabular}{|c|c|c|c|c|c|c|c|c|c|c|c|c|c|c|c|c|}
\hline \multirow{2}{*}{ Fungal Isolate } & \multirow{2}{*}{$N$} & \multicolumn{2}{|c|}{ Amphotericin B } & \multicolumn{2}{|c|}{ Fluconazole } & \multicolumn{2}{|c|}{ Voriconazole } & \multicolumn{2}{|c|}{ Itraconazole } & \multicolumn{2}{|c|}{ Posaconazole } & \multicolumn{2}{|c|}{ Caspofungin } & \multicolumn{2}{|c|}{ Micafungin } & \multirow{2}{*}{$\begin{array}{c}\text { Flucytosine } \\
\mathrm{S}\end{array}$} \\
\hline & & $\mathrm{S}$ & $\mathbf{R}$ & $\mathrm{s}$ & $\mathbf{I} / \mathbf{R}$ & $\mathrm{S}$ & $\mathbf{I} / \mathbf{R}$ & $\mathrm{s}$ & $\mathbf{I} / \mathbf{R}$ & $\mathrm{s}$ & $\mathbf{R}$ & $S$ & $\mathbf{I} / \mathbf{R}$ & $S$ & $\mathbf{I} / \mathbf{R}$ & \\
\hline Candida albicans, $n(\%)$ & 14 & $14(100)$ & 0 & $11(78.6)$ & $\begin{array}{l}\text { I: } 2 \text { (14.3); } \\
\text { R: 1 (7.1) }\end{array}$ & 11 (78.6) & I: $3(21.4)$ & $10(71.4)$ & $\begin{array}{l}\text { I: } 3 \text { (21.4); } \\
\text { R: } 1 \text { (7.1) }\end{array}$ & & & $13(92.9)$ & I: 1 (7.1) & $14(100)$ & 0 & \\
\hline C. tropicalis, $n(\%)$ & 10 & $10(100)$ & 0 & $10(100)$ & 0 & $10(100)$ & 0 & $10(100)$ & 0 & & & $8(80)$ & I: $2(20)$ & $7(70)$ & $\begin{array}{l}\text { I: } 2 \text { (20); R: } \\
\quad 1(10)\end{array}$ & \\
\hline C. parapsilosis, $n(\%)$ & 14 & $14(100)$ & 0 & $10(71.4)$ & $\begin{array}{l}\text { I: } 1 \text { (7.1); R: } \\
3 \text { (21.4) }\end{array}$ & 11 (78.6) & I: $3(21.4)$ & $10(71.4)$ & $\begin{array}{l}\text { I: } 3 \text { (21.4); } \\
\text { R: } 1 \text { (7.1) }\end{array}$ & & & $14(100)$ & 0 & $14(100)$ & 0 & \\
\hline C. guillermondii, $n(\%)$ & 2 & $2(100)$ & 0 & $1(50)$ & $1(50)$ & $2(100)$ & 0 & $2(100)$ & 0 & & & $2(100)$ & 0 & & $\begin{array}{l}\text { I: } 1(50) ; \mathrm{R}: \\
\quad 1(50)\end{array}$ & \\
\hline C. auris, $n(\%)$ & 2 & $2(100)$ & 0 & $2(100)$ & 0 & $2(100)$ & 0 & $2(100)$ & 0 & & & $2(100)$ & 0 & $2(100)$ & 0 & \\
\hline C. glabrata, $n(\%)$ & 8 & $8(100)$ & 0 & $8(100)$ & 0 & $3(37.5)$ & I: $5(62.5)$ & $5(62.5)$ & I: $3(37.5)$ & & & $4(50)$ & $\begin{array}{l}\text { I: } 1 \text { (12.5); } \\
\text { R:3 (37.5) }\end{array}$ & $4(50)$ & $\begin{array}{l}\text { I: } 1 \text { (12.5); } \\
\text { R: } 3 \text { (37.5) }\end{array}$ & \\
\hline Lodderomyces longisporus, $n(\%)$ & 1 & $1(100)$ & 0 & $1(100)$ & 0 & $1(100)$ & 0 & $1(100)$ & 0 & & & $1(100)$ & 0 & $1(100)$ & 0 & \\
\hline Cryptococcus neoformans, $n(\%)$ & 24 & $24(100)$ & 0 & $24(100)$ & 0 & $24(100)$ & 0 & & & & & & & & & $24(100)$ \\
\hline Aspergillus flavus, $n(\%)$ & 42 & $42(100)$ & 0 & & & $42(100)$ & 0 & $41(97.6)$ & $1(2.3)$ & $42(100)$ & 0 & $42(100)$ & 0 & $42(100)$ & 0 & \\
\hline A. fumigatus, $n(\%)$ & 43 & $43(100)$ & 0 & & & $43(100)$ & 0 & $42(97.6)$ & $1(2.3)$ & $43(100)$ & 0 & 41 & $2(4.7)$ & $43(100)$ & 0 & \\
\hline A. terreus, $n(\%)$ & 7 & $7(100)$ & 0 & & & $7(100)$ & 0 & $7(100)$ & 0 & $7(100)$ & 0 & $7(100)$ & 0 & $7(100)$ & 0 & \\
\hline A. niger, $n(\%)$ & 8 & $8(100)$ & 0 & & & $8(100)$ & 0 & $7(87.5)$ & $1(12.5)$ & $8(100)$ & 0 & $8(100)$ & 0 & $8(100)$ & 0 & \\
\hline Rhizopus arrhizus, $n$ (\%) & 54 & $54(100)$ & 0 & & & $54(100)$ & 0 & $42(77.7)$ & $12(22.2)$ & $39(72.2)$ & $15(27.8)$ & & & & & \\
\hline R. microsporus, $n(\%)$ & 30 & $30(100)$ & 0 & & & $30(100)$ & 0 & $27(90)$ & $3(10)$ & $23(76.6)$ & $7(23.3)$ & & & & & \\
\hline R. pusillus, $n(\%)$ & 13 & $13(100)$ & 0 & & & $13(100)$ & 0 & $13(100)$ & 0 & $13(100)$ & 0 & & & & & \\
\hline Lichtheimia corymbifera, $n(\%)$ & 1 & $1(100)$ & 0 & & & $1(100)$ & 0 & $1(100)$ & 0 & $1(100)$ & 0 & & & & & \\
\hline L. ramosa, $n(\%)$ & 4 & $4(100)$ & 0 & & & $4(100)$ & 0 & $4(100)$ & 0 & $4(100)$ & 0 & & & & & \\
\hline Apophysomyces variabilis, $n(\%)$ & 1 & $1(100)$ & 0 & & & $1(100)$ & 0 & $1(100)$ & 0 & $1(100)$ & 0 & & & & & \\
\hline Mucor circinelloides, $n(\%)$ & 1 & $1(100)$ & 0 & & & $1(100)$ & 0 & 0 & $1(100)$ & $1(100)$ & 0 & & & & & \\
\hline Conidiobolus coronatus, $n(\%)$ & 3 & $3(100)$ & 0 & & & $3(100)$ & 0 & $3(100)$ & 0 & $3(100)$ & 0 & & & & & \\
\hline Taleromyces marneffi, $n(\%)$ & 1 & $1(100)$ & 0 & & & $1(100)$ & 0 & $1(100)$ & 0 & $1(100)$ & 0 & & & & & \\
\hline
\end{tabular}

Note: $N$, total number of isolates; $\mathrm{S}$, susceptible; I, intermediate; $\mathrm{R}$, resistant. 


\section{Discussion}

We describe epidemiology, predisposing factors, antifungal susceptibility patterns, and outcome in invasive fungal infections (IFIs) from a tertiary care center in India. Overall, we found a significant incidence of IFIs in our mixed but high-risk cohort of patients. The observed rate of $7.6 \%$ in this study is in accordance with previously published literature of IFIs in antifungal-naïve population [5-11,30-34].

In our mixed cohort of patients, invasive aspergillosis (IA) $(40.7 \%, 103 / 253)$ emerged as the most common IFI, followed by mucormycosis $(24.5 \%, 62 / 253)$. This is in accordance with studies conducted in the hematological patient population $[34,35]$ and in contrast with reports of invasive candidiasis being the most common IFI [13,36]. Traditional underlying conditions noted in this study were dominated by prolonged exposure to corticosteroids (24.5\%), diabetes mellitus (23.7\%), and hematological malignancies (19.8\%). Among other predisposing conditions, chronic kidney disease (CKD) (34.4\%) and pulmonary manifestations (30.8\%) were the most prevalent. These data are consistent with the range of patient populations affected inside and outside of traditional high-risk groups [7,37-43] Contrary to global data, $22.1 \%$ of IFI cases noted in this study were from the ICUs, with a predominance of $55.3 \%$ IA followed by $33.9 \%$ IC and the remaining $10.7 \%$ of mucormycosis cases $[44,45]$.

In this study, species identification revealed that Candida albicans and Candida parapsilosis were the most common cause of invasive candidiasis (IC), which is incongruent with the listing of Candida tropicalis as the most common cause of IC from India [46,47]. However, fungal isolations of Cryptococcus neoformans, Aspergillus flavus, and Rhizopus arrhizus were in accordance with previously published literature on respective IFIs [27,48-50].

Similar to global data in our invasive candidiasis isolates, azole resistance was noted in C. albicans, C. parapsilosis, and C. glabrata, whereas echinocandin resistance was noted in C. albicans and C. glabrata [51-55]. It is significant that for echinocandins, in vitro susceptibility tested resistance is known to translate into treatment failures owing to FKS mutations $[52,53,56]$. Previous invasive candidiasis data from our center listed $<6 \%$ resistance to fluconazole and 100\% sensitivity to amphotericin B [57]. Global data support the CLSI C. albicans clinical breakpoints for fluconazole, whereas lacking the similar acceptance for C. glabrata, the most isolates of this non-albicans species fall in the intermediate category [17]. To overcome these shortcomings, susceptibility testing research has broadened. Natural oils from algae such as Ruta graveolans or north Sardinia plants have been evaluated for their efficacy (fungistatic and fungicidal). They have been found active against multidrug-resistant Candida sp. [58,59].

Although once known to be rare, cryptococcosis has occurred at a high frequency in India in the past two decades, as envisaged in a recent multicenter study [50]. It is one of the AIDS-defining infections and is responsible for about 15\% of AIDS-related deaths [60]. However, in the current study, 50\% of cases were seen with renal involvement, and only 29.4\% were AIDS-related. The decrease in AIDS-related secondary cryptococcal infection may be owing to highly active antiretroviral therapy (HAART) therapy [60].

For Cryptococcosis, the drugs of choice are described in detail [19]. Amphotericin B (and its lipid formulations) with flucytosine is indicated as induction therapy in HIVinfected individuals, organ transplant recipients, non-HIV, and non-transplant patients, with differences in dosage and duration. The maintenance and consolidation therapy is fluconazole. For patients with CD4 count $>100$ cells $/ \mu \mathrm{L}$ and undetectable viral load for $>3$ months, a minimum of 1 year of antifungal therapy is recommended [19]. From India, high MICs against fluconazole and flucytosine have been reported [61-63]. However, from our center in the current and another multicenter study [19], 100\% sensitivity was noted for all the drugs.

In Western countries, local epidemiology highlights the predominance of $A$. fumigatus in invasive aspergillosis (IA) cases [64,65], whereas from India, A.flavus is most commonly isolated. Voriconazole is the drug of choice for primary therapy (especially with cases of invasive pulmonary aspergillosis) [20,66]. However, triazole (itraconazole, voriconazole, 
and posaconazole) drug resistance has been previously reported $[64,67,68]$. In this study, only three strains showed high MICs to itraconazole, of which one was A. fumigatus. In the Western world, Aspergillus fumigatus azole resistance (ARAF) has been extensively researched for its clinical implications [69-72], whereas from India, there are few sporadic reports of clinical and environmental ARAF strains [28,73-75].

Another life-threatening IFI that was noted in high numbers in this study was mucormycosis. It presented in its most common form, rhino-orbital, and with the usual predisposing conditions of renal involvement and ketoacidosis [48]. The increasing trends of this infection hint towards breakthrough infections [76,77]. Antifungal treatment strategies are generally associated with surgical intervention for these cases. The focus is on the roles of amphotericin B formulations, posaconazole, combination therapies, and newer therapeutic approaches [78]. It is important to identify the genus, or if possible the species, since Cuninghamella, Lichtheimia, and Rhizopus oryzae can be resistant to posaconazole, which usually shows susceptible MIC profiles [79,80]. The standard treatment is liposomal amphotericin B dose according to the localization and extent of infection. The role of posaconazole is that it can be used as salvage therapy along with amphotericin B [80,81]. Incongruent with amphotericin B susceptibility data from India, in this study all strains were susceptible [82-84]. However, about 70\% of Rhizopus species were susceptible to posaconazole, which is similar to previously published data [82-84].

Novel antifungal therapies and strategies can aid in the management of IFIs. In highrisk patients (neutropenic, etc.), antifungal prophylaxis is also recommended. However, the benefits associated with antifungal therapy (prophylactic/empirical) need to be evaluated with respect to local epidemiology and cost effectiveness. The treatment modalities are still unavailable/unaffordable to many patients in a developing nation such as ours.

The study was limited by its clinical suspicion inclusion bias and unexpectedly low numbers of probable IFIs, which may be due to the lack of invasive sampling owing to the poor condition of patients. There was one Taleromyces marneffi recovered from an AIDS patient, limiting the overall picture of the burden of rare pathogens among these infections.

\section{Conclusion}

In conclusion, the local epidemiology of IFIs in this study was significantly different from elsewhere. The predictors of infection or mortality were found similar to global data. However, these considerations underscore the importance of understanding both the epidemiology and resistance profile of the invasive fungal isolates that are commonly seen in both immunocompromised and immunocompetent populations. An active surveillance of invasive fungal infections, along with multidrug susceptibility testing of isolates to monitor the extent of the problem and develop feasible local diagnostic algorithms, will provide the database that might aid in future treatments to limit the emergence of resistance and alleviate the fatality rate.

Supplementary Materials: The following is available online at https:/ /www.mdpi.com/article/10.3 390/jof8010033/s1, Figure S1: Fungal species distribution in samples from IFI cases $(n=253)$.

Author Contributions: Conceptualization, I.X. and Y.D.; methodology, I.X. and Y.D.; software, Y.D.; formal analysis, Y.D.; patient investigation, M.M., R.S., S.B., R.K., V.P.J. and S.M.; experimentation, Y.D., M.P., J.A., J.S. and A.I.; resources, I.X.; data curation, I.X. and Y.D.; writing-original draft preparation, Y.D.; writing-review and editing, I.X. and Y.D.; supervision, I.X., G.S., M.M., R.S., S.B., R.K., V.P.J. and S.M; project administration, I.X.; funding acquisition, Y.D. All authors have read and agreed to the published version of the manuscript.

Funding: This research was funded by Indian Council of Medical Research [OMI/15/2014-ECD-I]. 
Institutional Review Board Statement: The study was conducted according to the guidelines of the Declaration of Helsinki, and the study was approved by the ethics committee of the institute i.e., All India Institute of Medical Sciences, New Delhi, India (Ref no. IEC/NP-25/2014RP-10/2014, OP3/09.02.2017). The detailed procedure was as per institute guidelines: http:/ /www.aiims.edu/aiims/ academic/ethics-committee/forms\%20in\%20pdf/IEC/Format_of_Institution_Ethics_Committee_15 032012.pdf (accessed on 16 January 2017).

Informed Consent Statement: The consent forms for minor/incapable participants were obtained by their LAR, i.e., legally accepted representatives (example: mother, father, children, or grandparents)

Acknowledgments: The authors are grateful to patients and their relatives for providing consent for the study and for their cooperation. Immaculata Xess received funds for this study from the Indian Council of Medical Research (ICMR), File No. OMI/15/2014-ECD-I.

Conflicts of Interest: The authors declare no conflict of interest.

\section{References}

1. Hsu, L.; Lee, D.; Yeh, S.; Bhurani, D.; Khanh, B.; Low, C.; Norasetthada, L.; Chan, T.; Kwong, Y.; Vaid, A.; et al. Epidemiology of invasive fungal diseases among patients with haematological disorders in the Asia-Pacific: A prospective observational study. Clin. Microbiol. Infect. 2015, 21, 594.e7-594.e11. [CrossRef] [PubMed]

2. Slavin, M.A.; Chakrabarti, A. Opportunistic fungal infections in the Asia-Pacific region. Med. Mycol. 2012, 50, 18-25. [CrossRef] [PubMed]

3. Hoenigl, M. Invasive fungal disease complicating COVID-19: When it rains it pours. Clin. Infect. Dis. 2020, 73, e1645-e1648. [CrossRef] [PubMed]

4. Firacative, C. Invasive fungal disease in humans: Are we aware of the real impact? Mem. Inst. Oswaldo Cruz 2020, 115, e200430. [CrossRef]

5. Nganthavee, V.; Phutthasakda, W.; Atipas, K.; Tanpong, S.; Pungprasert, T.; Dhirachaikulpanich, D.; Krithin, S.; Tanglitanon, S.; Jutidamronphang, W.; Owattanapanich, W.; et al. High incidence of invasive fungal infection during acute myeloid leukemia treatment in a resource-limited country: Clinical risk factors and treatment outcomes. Support. Care Cancer 2019, 27, 3613-3622. [CrossRef]

6. Tang, J.-L.; Kung, H.-C.; Lei, W.-C.; Yao, M.; Wu, U.-I.; Hsu, S.-C.; Lin, C.-T.; Li, C.-C.; Wu, S.-J.; Hou, H.-A.; et al. High incidences of invasive fungal infections in acute Myeloid Leukemia patients receiving induction chemotherapy without systemic antifungal Prophylaxis: A prospective observational study in Taiwan. PLoS ONE 2015, 10, e0128410. [CrossRef]

7. Lien, M.-Y.; Chou, C.-H.; Lin, C.-C.; Bai, L.-Y.; Chiu, C.-F.; Yeh, S.-P.; Ho, M.-W. Epidemiology and risk factors for invasive fungal infections during induction chemotherapy for newly diagnosed acute myeloid leukemia: A retrospective cohort study. PLoS ONE 2018, 13, e0197851. [CrossRef]

8. Gheith, S.; Saghrouni, F.; Bannour, W.; Ben Youssef, Y.; Khelif, A.; Normand, A.-C.; Ben Saïd, M.; Piarroux, R.; Njah, M.; Ranque, S. Characteristics of invasive Aspergillosis in Neutropenic Haematology patients (Sousse, Tunisia). Mycopathologia 2014, 177, 281-289. [CrossRef]

9. Hammond, S.P.; Marty, F.M.; Bryar, J.M.; DeAngelo, D.J.; Baden, L.R. Invasive fungal disease in patients treated for newly diagnosed acute leukemia. Am. J. Hematol. 2010, 85, 695-699. [CrossRef]

10. Chen, C.Y.; Sheng, W.H.; Tien, F.M.; Lee, P.C.; Huang, S.Y.; Tang, J.L.; Tsay, W.; Tien, H.F.; Hsueh, P.R. Clinical characteristics and treatment outcomes of pulmonary invasive fungal infection among adult patients with hematological malignancy in a medical centre in Taiwan, 2008-2013. J. Microbiol. Immunol. Infect. 2020, 53, 106-114. [CrossRef] [PubMed]

11. Kumar, J.; Singh, A.; Seth, R.; Xess, I.; Jana, M.; Kabra, S.K. Prevalence and predictors of invasive fungal infections in children with persistent febrile neutropenia treated for acute leukemia-A prospective study. Indian J. Pediatrics 2018, 85, 1090-1095. [CrossRef]

12. Jacobs, S.; Wengenack, N.L.; Walsh, T.J. Non-Aspergillus Hyaline molds: Emerging causes of sino-pulmonary fungal infections and other invasive mycoses. Semin. Respir. Crit. Care Med. 2020, 41, 115-130. [CrossRef] [PubMed]

13. Li, Y.; Gao, Y.; Niu, X.; Wu, Y.; Du, Y.; Yang, Y.; Qi, R.; Chen, H.; Gao, X.; Song, B.; et al. A 5-year review of invasive fungal infection at an academic medical center. Front. Cell. Infect. Microbiol. 2020, 10, 553648. [CrossRef] [PubMed]

14. Lamoth, F.; Lockhart, S.R.; Berkow, E.L.; Calandra, T. Changes in the epidemiological landscape of invasive candidiasis. J. Antimicrob. Chemother. 2018, 73, i4-i13. [CrossRef] [PubMed]

15. Hendrickson, J.; Hu, C.; Aitken, S.L.; Beyda, N. Antifungal resistance: A concerning trend for the present and future. Curr. Infect. Dis. Rep. 2019, 21, 47. [CrossRef]

16. Wall, G.; Lopez-Ribot, J.L. Current antimycotics, new prospects, and future approaches to antifungal therapy. Antibiotics 2020, 9 , 445. [CrossRef]

17. Lamoth, F.; Lewis, R.E.; Kontoyiannis, D.P. Role and interpretation of antifungal susceptibility testing for the management of invasive fungal infections. J. Fungi 2020, 7, 17. [CrossRef] 
18. Pappas, P.G.; Kauffman, C.A.; Andes, D.R.; Clancy, C.J.; Marr, K.A.; Ostrosky-Zeichner, L.; Reboli, A.C.; Schuster, M.G.; Vazquez, J.A.; Walsh, T.J. Clinical practice guideline for the management of candidiasis: 2016 update by the Infectious Diseases Society of America. Clin. Infect. Dis. 2016, 62, e1-e50. [CrossRef]

19. Perfect, J.R.; Dismukes, W.E.; Dromer, F.; Goldman, D.L.; Graybill, J.R.; Hamill, R.J.; Harrison, T.S.; Larsen, R.A.; Lortholary, O.; Nguyen, M.-H.; et al. Clinical practice guidelines for the management of cryptococcal disease: 2010 update by the Infectious Diseases Society of America. Clin. Infect. Dis. 2010, 50, 291-322. [CrossRef]

20. Patterson, T.F.; Thompson, G.R., III; Denning, D.W.; Fishman, J.A.; Hadley, S.; Herbrecht, R.; Kontoyiannis, D.P.; Marr, K.A.; Morrison, V.A.; Nguyen, M.H. Practice guidelines for the diagnosis and management of aspergillosis: 2016 update by the Infectious Diseases Society of America. Clin. Infect. Dis. 2016, 63, e1-e60. [CrossRef]

21. Cornely, O.A.; Alastruey-Izquierdo, A.; Arenz, D.; Chen, S.C.; Dannaoui, E.; Hochhegger, B.; Hoenigl, M.; Jensen, H.E.; Lagrou, K.; Lewis, R.E.; et al. Global guideline for the diagnosis and management of mucormycosis: An initiative of the European Confederation of Medical Mycology in cooperation with the Mycoses Study Group Education and Research Consortium. Lancet Infect. Dis. 2019, 19, e405-e421. [CrossRef]

22. De Pauw, B.; Walsh, T.J.; Donnelly, J.P.; Stevens, D.A.; Edwards, J.E.; Calandra, T.; Pappas, P.G.; Maertens, J.; Lortholary, O.; Kauffman, C.A. Revised definitions of invasive fungal disease from the European organization for research and treatment of cancer/invasive fungal infections cooperative group and the national institute of allergy and infectious diseases mycoses study group (EORTC/MSG) consensus group. Clin. Infect. Dis. 2008, 46, 1813-1821. [PubMed]

23. Blot, S.I.; Taccone, F.S.; Abeele, A.-M.V.D.; Bulpa, P.; Meersseman, W.; Brusselaers, N.; Dimopoulos, G.; Paiva, J.A.; Misset, B.; Rello, J.; et al. A clinical algorithm to diagnose invasive pulmonary Aspergillosis in critically Ill patients. Am. J. Respir. Crit. Care Med. 2012, 186, 56-64. [CrossRef]

24. Donnelly, J.P.; Chen, S.C.; Kauffman, C.A.; Steinbach, W.J.; Baddley, J.W.; Verweij, P.E.; Clancy, C.J.; Wingard, J.R.; Lockhart, S.R.; Groll, A.H. Revision and update of the consensus definitions of invasive fungal disease from the European Organization for Research and Treatment of Cancer and the Mycoses Study Group Education and Research Consortium. Clin. Infect. Dis. 2020, 71, 1367-1376. [CrossRef]

25. CLSI. Performance Standards for Antifungal Susceptibility Testing of Yeasts, 2nd ed.; Clinical and Laboratory Standards Institute: Wayne, PA, USA, 2020; Volume M60ed2.

26. Clinical Laboratory Standards Institute. Reference Method for Broth Dilution Antifungal Susceptibility Testing of Filamentous Fungi. Approved Standard, 2nd ed.; Clinical and Laboratory Standards Institute: Wayne, PA, USA, 2008; Volume M38-A2.

27. Xess, I.; Pandey, M.; Dabas, Y.; Agarwal, R.; Das, S.; Srivastava, P.M.V.; Thakur, R.; Sharma, S.; Mani, P.; Biswas, A.; et al. Multilocus sequence typing of clinical isolates of Cryptococcus from India. Mycopathologia 2021, 186, 199-211. [CrossRef]

28. Dabas, Y.; Xess, I.; Bakshi, S.; Mahapatra, M.; Seth, R. Emergence of Azole-Resistant Aspergillus fumigatus from immunocompromised hosts in India. Antimicrob. Agents Chemother. 2018, 62, 02264-17. [CrossRef] [PubMed]

29. Almyroudis, N.G.; Sutton, D.A.; Fothergill, A.W.; Rinaldi, M.G.; Kusne, S. In vitro susceptibilities of 217 clinical isolates of Zygomycetes to conventional and new antifungal agents. Antimicrob. Agents Chemother. 2007, 51, 2587-2590. [CrossRef]

30. Kim, G.Y.; Burns, J.; Freyer, C.W.; Hamilton, K.W.; Frey, N.V.; Gill, S.I.; Hexner, E.O.; Luger, S.M.; Mangan, J.K.; Martin, M.E.; et al. Risk of invasive fungal infections in patients with high-risk MDS and AML receiving hypomethylating agents. Am. J. Hematol. 2020, 95, 792-798. [CrossRef] [PubMed]

31. Liu, Y.-C.; Chien, S.-H.; Fan, N.-W.; Hu, M.-H.; Gau, J.-P.; Liu, C.-J.; Yu, Y.-B.; Liu, C.-Y.; Hsiao, L.-T.; Liu, J.-H.; et al. Incidence and risk factors of probable and proven invasive fungal infection in adult patients receiving allogeneic hematopoietic stem cell transplantation. J. Microbiol. Immunol. Infect. 2016, 49, 567-574. [CrossRef]

32. Şular, F.-L.; Szekely, E.; Cristea, V.C.; Dobreanu, M. Invasive fungal infection in Romania: Changing incidence and epidemiology during six years of surveillance in a tertiary hospital. Mycopathologia 2018, 183, 967-972. [CrossRef] [PubMed]

33. Lien, M.-Y.; Yeh, S.-P.; Gau, J.-P.; Wang, P.-N.; Li, S.-S.; Dai, M.-S.; Chen, T.C.; Hsieh, P.-Y.; Chiou, L.-W.; Ko, B.S.; et al. High rate of invasive fungal infections after non-T cell depleted haploidentical allo-HSCT even under antifungal prophylaxis. Bone Marrow Transplant. 2021, 56, 1-4. [CrossRef]

34. Harrison, N.; Mitterbauer, M.; Tobudic, S.; Kalhs, P.; Rabitsch, W.; Greinix, H.T.; Burgmann, H.; Willinger, B.; Presterl, E.; Forstner, $\mathrm{C}$. Incidence and characteristics of invasive fungal diseases in allogeneic hematopoietic stem cell transplant recipients: A retrospective cohort study. BMC Infect. Dis. 2015, 15, 584. [CrossRef]

35. Vazquez, A.J.; Tovar-Torres, M.P.; Hingwe, A.; Cheema, F.; Welch, V.L.; Ford, K.D. The changing epidemiology of invasive Aspergillosis in the non-traditional host: Risk factors and outcomes. Pulm. Crit. Care Med. 2016, 1, 5. [CrossRef]

36. Neofytos, D.; Fishman, J.; Horn, D.; Anaissie, E.; Chang, C.-H.; Olyaei, A.; Pfaller, M.; Steinbach, W.; Webster, K.; Marr, K. Epidemiology and outcome of invasive fungal infections in solid organ transplant recipients. Transpl. Infect. Dis. 2010, 12, 220-229. [CrossRef] [PubMed]

37. Zhang, H.; Zhu, A. Emerging invasive fungal infections: Clinical features and controversies in diagnosis and treatment processes. Infect. Drug Resist. 2020, 13, 607-615. [CrossRef]

38. Shariati, A.; Moradabadi, A.; Chegini, Z.; Khoshbayan, A.; Didehdar, M. An overview of the management of the most important invasive fungal infections in patients with blood malignancies. Infect. Drug Resist. 2020, 13, 2329-2354. [CrossRef] [PubMed]

39. Patel, M.H.; Patel, R.D.; Vanikar, A.V.; Kanodia, K.V.; Suthar, K.S.; Nigam, L.K.; Patel, H.V.; Patel, A.H.; Kute, V.B.; Trivedi, H.L. Invasive fungal infections in renal transplant patients: A single center study. Ren. Fail. 2017, 39, 294-298. [CrossRef] 
40. Chang, A.; Musk, M.; Lavender, M.; Wrobel, J.; Yaw, M.; Lawrence, S.; Chirayath, S.; Boan, P. Epidemiology of invasive fungal infections in lung transplant recipients in Western Australia. Transpl. Infect. Dis. 2019, 21, e13085. [CrossRef]

41. Boroujeni, Z.B.; Shamsaei, S.; Yarahmadi, M.; Getso, M.I.; Khorashad, A.S.; Haghighi, L.; Raissi, V.; Zareei, M.; Mohammadzade, A.S.; Moqarabzadeh, V.; et al. Distribution of invasive fungal infections: Molecular epidemiology, etiology, clinical conditions, diagnosis and risk factors: A 3-year experience with 490 patients under intensive care. Microb. Pathog. 2021, 152, 104616. [CrossRef]

42. Bajpai, V.K.; Khan, I.; Shukla, S.; Kumar, P.; Rather, I.A.; Park, Y.-H.; Huh, Y.S.; Han, Y.-K. Invasive fungal infections and their epidemiology: Measures in the clinical scenario. Biotechnol. Bioprocess. Eng. 2019, 24, 436-444. [CrossRef]

43. Enoch, D.A.; Yang, H.; Aliyu, S.H.; Micallef, C. The changing epidemiology of invasive fungal infections. In Methods in Molecular Biology; Springer: New York, NY, USA, 2017; Volume 1508, pp. 17-65.

44. Paramythiotou, E.; Frantzeskaki, F.; Flevari, A.; Armaganidis, A.; Dimopoulos, G. Invasive fungal infections in the ICU: How to approach, how to treat. Molecules 2014, 19, 1085-1119. [CrossRef] [PubMed]

45. Montagna, M.T.; Caggiano, G.; Lovero, G.; De Giglio, O.; Coretti, C.; Cuna, T.; Iatta, R.; Giglio, M.; Dalfino, L.; Bruno, F.; et al. Epidemiology of invasive fungal infections in the intensive care unit: Results of a multicenter Italian survey (AURORA Project). Infection 2013, 41, 645-653. [CrossRef] [PubMed]

46. Xess, I.; Jain, N.; Hasan, F.; Mandal, P.; Banerjee, U. Epidemiology of Candidemia in a Tertiary Care Centre of North India: 5-year study. Infection 2007, 35, 256-259. [CrossRef]

47. Chakrabarti, A.; Sood, P.; Rudramurthy, S.; Chen, S.; Kaur, H.; Capoor, M.; Chhina, D.; Rao, R.; Eshwara, V.K.; Xess, I.; et al. Incidence, characteristics and outcome of ICU-acquired candidemia in India. Intensiv. Care Med. 2014, 41, 285-295. [CrossRef] [PubMed]

48. Patel, A.; Kaur, H.; Xess, I.; Michael, J.; Savio, J.; Rudramurthy, S.; Singh, R.; Shastri, P.; Umabala, P.; Sardana, R.; et al. A multicentre observational study on the epidemiology, risk factors, management and outcomes of mucormycosis in India. Clin. Microbiol. Infect. 2020, 26, 944.e9-944.e15. [CrossRef]

49. Rudramurthy, S.M.; Paul, R.A.; Chakrabarti, A.; Mouton, J.W.; Meis, J.F. Invasive Aspergillosis by Aspergillus flavus: Epidemiology, diagnosis, antifungal resistance, and management. J. Fungi 2019, 5, 55. [CrossRef]

50. Jain, N.; Wickes, B.L.; Keller, S.M.; Fu, J.; Casadevall, A.; Jain, P.; Ragan, M.A.; Banerjee, U.; Fries, B.C. Molecular epidemiology of clinical Cryptococcus neoformans strains from India. J. Clin. Microbiol. 2005, 43, 5733-5742. [CrossRef]

51. Coste, A.T.; Kritikos, A.; Li, J.; Khanna, N.; Goldenberger, D.; Garzoni, C.; Zehnder, C.; Boggian, K.; Neofytos, D.; Riat, A.; et al. Emerging echinocandin-resistant Candida albicans and glabrata in Switzerland. Infection 2020, 48, 761-766. [CrossRef]

52. Beyda, N.D.; John, J.; Kilic, A.; Alam, M.J.; Lasco, T.M.; Garey, K.W. FKS mutant Candida glabrata: Risk factors and outcomes in patients with Candidemia. Clin. Infect. Dis. 2014, 59, 819-825. [CrossRef]

53. Shields, R.K.; Nguyen, M.H.; Press, E.G.; Cumbie, R.; Driscoll, E.; Pasculle, A.W.; Clancy, C.J. Rate of FKS Mutations among consecutive Candida isolates causing bloodstream infection. Antimicrob. Agents Chemother. 2016, 60, 1954. [CrossRef]

54. Jia, X.; Li, C.; Cao, J.; Wu, X.; Zhang, L. Clinical characteristics and predictors of mortality in patients with candidemia: A six-year retrospective study. Eur. J. Clin. Microbiol. Infect. Dis. 2018, 37, 1717-1724. [CrossRef]

55. Xiao, M.; Chen, S.C.; Kong, F.; Xu, X.L.; Yan, L.; Kong, H.S.; Fan, X.; Hou, X.; Cheng, J.W.; Zhou, M.L. Dis-tribution and antifungal susceptibility of Candida species causing candidemia in China: An update from the CHIF-NET study. J. Infect. Dis. 2020, 221, S139-S147. [CrossRef]

56. Shields, R.K.; Nguyen, M.H.; Press, E.G.; Updike, C.L.; Clancy, C.J. Caspofungin MICs correlate with treatment outcomes among patients with Candida glabrata invasive Candidiasis and prior Echinocandin exposure. Antimicrob. Agents Chemother. 2013, 57, 3528-3535. [CrossRef]

57. Singh, R.I.; Xess, I.; Mathur, P.; Behera, B.; Gupta, B.; Misra, M.C. Epidemiology of candidaemia in critically ill trauma patients: Experiences of a level I trauma centre in North India. J. Med. Microbiol. 2010, 60, 342-348. [CrossRef]

58. Donadu, M.; Peralta-Ruiz, Y.; Usai, D.; Maggio, F.; Molina-Hernandez, J.; Rizzo, D.; Bussu, F.; Rubino, S.; Zanetti, S.; Paparella, A.; et al. Colombian essential oil of Ruta graveolens against Nosocomial Antifungal Resistant Candida strains. J. Fungi 2021, 7, 383. [CrossRef] [PubMed]

59. Donadu, M.; Usai, D.; Marchetti, M.; Usai, M.; Mazzarello, V.; Molicotti, P.; Montesu, M.; Delogu, G.; Zanetti, S. Antifungal activity of oils macerates of North Sardinia plants against Candida species isolated from clinical patients with candidiasis. Nat. Prod. Res. 2020, 34, 3280-3284. [CrossRef] [PubMed]

60. Nyazika, T.K.; Tatuene, J.K.; Kenfak-Foguena, A.; Verweij, P.; Meis, J.F.; Robertson, V.J.; Hagen, F. Epidemiology and aetiologies of cryptococcal meningitis in Africa, 1950-2017: Protocol for a systematic review. BMJ Open 2018, 8, e20654. [CrossRef]

61. Datta, K.; Jain, N.; Sethi, S.; Rattan, A.; Casadevall, A.; Banerjee, U. Fluconazole and itraconazole susceptibility of clinical isolates of Cryptococcus neoformans at a tertiary care centre in India: A need for care. J. Antimicrob. Chemo-Ther. 2003, 52, 683-686. [CrossRef] [PubMed]

62. Capoor, M.R.; Mandal, P.; Deb, M.; Aggarwal, P.; Banerjee, U. Current scenario of cryptococcosis and antifungal susceptibility pattern in India: A cause for reappraisal. Mycoses 2008, 51, 258-265. [CrossRef]

63. Chowdhary, A.; Randhawa, H.S.; Sundar, G.; Kathuria, S.; Prakash, A.; Khan, Z.; Sun, S.; Xu, J. In vitro antifungal susceptibility profiles and genotypes of 308 clinical and environmental isolates of Cryptococcus neoformans var. grubii and Cryptococcus gattii serotype B from north-western. India. J. Med. Microbiol. 2011, 60, 961-967. [CrossRef] [PubMed] 
64. Lestrade, P.P.; Van Der Velden, W.J.F.M.; Bouwman, F.; Stoop, F.J.; Blijlevens, A.N.M.; Melchers, W.J.G.; Verweij, P.E.; Donnelly, J.P. Epidemiology of invasive aspergillosis and triazole-resistant Aspergillus fumigatus in patients with haematological malignancies: A single-centre retrospective cohort study. J. Antimicrob. Chemother. 2018, 73, 1389-1394. [CrossRef] [PubMed]

65. Lockhart, S.R.; Beer, K.; Toda, M. Azole-Resistant Aspergillus fumigatus: What you need to know. Clin. Microbiol. Newsl. 2020, 42, 1-6. [CrossRef]

66. Walsh, T.J.; Anaissie, E.J.; Denning, D.; Herbrecht, R.; Kontoyiannis, D.P.; Marr, K.A.; Morrison, V.A.; Segal, B.H.; Steinbach, W.J.; Stevens, D.A.; et al. Treatment of Aspergillosis: Clinical practice guidelines of the Infectious Diseases Society of America. Clin. Infect. Dis. 2008, 46, 327-360. [CrossRef]

67. Garcia-Rubio, R.; Cuenca-Estrella, M.; Mellado, E. Triazole resistance in Aspergillus species: An emerging problem. Drugs 2017, 77, 599-613. [CrossRef]

68. Rivero-Menendez, O.; Alastruey-Izquierdo, A.; Mellado, E.; Cuenca-Estrella, M. Triazole Resistance in Aspergillus spp.: A Worldwide Problem? J. Fungi 2016, 2, 21. [CrossRef]

69. Alanio, A.; Sitterlé, E.; Liance, M.; Farrugia, C.; Foulet, F.; Botterel, F.; Hicheri, Y.; Cordonnier, C.; Costa, J.M.; Bretagne, S. Low prevalence of resistance to azoles in Aspergillus fumigatus in a French cohort of patients treated for haematological malignancies. J. Antimicrob. Chemother. 2011, 66, 371-374. [CrossRef]

70. Bader, O.; Weig, M.; Reichard, U.; Lugert, R.; Kuhns, M.; Christner, M.; Held, J.; Peter, S.; Schumacher, U.; Buchheidt, D.; et al. cyp51A-Based Mechanisms of Aspergillus fumigatus Azole Drug Resistance Present in Clinical Samples from Germany. Antimicrob. Agents Chemother. 2013, 57, 3513-3517. [CrossRef]

71. Van Ingen, J.; Van Der Lee, H.A.L.; Rijs, A.J.M.M.; Snelders, E.; Melchers, W.; Verweij, P.E. High-level pan-azole-resistant Aspergillosis: TABLE 1. J. Clin. Microbiol. 2015, 53, 2343-2345. [CrossRef]

72. Fuhren, J.; Voskuil, W.S.; Boel, C.H.E.; Haas, P.J.A.; Hagen, F.; Meis, J.F.; Kusters, J.G. High prevalence of azole resistance in Aspergillus fumigatus isolates from high-risk patients. J. Antimicrob. Chemother. 2015, 70, 2894-2898. [CrossRef] [PubMed]

73. Chowdhary, A.; Kathuria, S.; Xu, J.; Sharma, C.; Sundar, G.; Singh, P.K.; Gaur, S.N.; Hagen, F.; Klaassen, C.H.; Meis, J.F. Clonal expansion and emergence of environmental multiple-triazole-resistant Aspergillus fumigatus strains carrying the TR34/L98H mutations in the cyp 51A gene in India. PLoS ONE 2012, 7, e52871. [CrossRef]

74. Chowdhary, A.; Sharma, C.; Kathuria, S.; Hagen, F.; Meis, J.F. Azole-resistant Aspergillus fumigatus with the environmental TR46/Y121F/T289A mutation in India. J. Antimicrob. Chemother. 2014, 69, 555-557. [CrossRef]

75. Chowdhary, A.; Sharma, C.; Kathuria, S.; Hagen, F.; Meis, J. Prevalence and mechanism of triazole resistance in Aspergillus fumigatus in a referral chest hospital in Delhi, India and an update of the situation in Asia. Front. Microbiol. 2015, 6, 428. [CrossRef] [PubMed]

76. Axell-House, D.B.; Wurster, S.; Jiang, Y.; Kyvernitakis, A.; Lewis, R.E.; Tarrand, J.J.; Raad, I.I.; Kontoyiannis, D.P. Breakthrough mucormycosis developing on mucorales-active antifungals portrays a poor prognosis in patients with hematologic cancer. $J$. Fungi 2021, 7, 217. [CrossRef] [PubMed]

77. Skiada, A.; Pavleas, I.; Drogari-Apiranthitou, M. Epidemiology and diagnosis of mucormycosis: An update. J. Fungi 2020, 6, 265. [CrossRef] [PubMed]

78. Pagano, L.; Cornely, O.A.; Busca, A.; Caira, M.; Cesaro, S.; Gasbarrino, C.; Girmenia, C.; Heinz, W.J.; Herbrecht, R.; Lass-Flörl, C. Combined antifungal approach for the treatment of invasive mucormycosis in patients with hematologic diseases: A report from the SEIFEM and FUNGISCOPE registries. Haematologica 2013, 98, e127. [CrossRef] [PubMed]

79. Garcia-Hermoso, D.; Alanio, A.; Lortholary, O.; Dromer, F. Agents of systemic and subcutaneous Mucormycosis and Entomophthoromycosis. In Clinical Microbiology Procedures Handbook; American Society for Microbiology: Washington, DC, USA, 2015; pp. 2087-2108.

80. Chen, S.C.-A.; Playford, E.G.; Sorrell, T.C. Antifungal therapy in invasive fungal infections. Curr. Opin. Pharmacol. 2010, 10, 522-530. [CrossRef] [PubMed]

81. Brunet, K.; Rammaert, B. Mucormycosis treatment: Recommendations, latest advances, and perspectives. J. Med. Mycol. 2020, 30, 101007. [CrossRef] [PubMed]

82. Bala, K.; Chander, J.; Handa, U.; Punia, R.S.; Attri, A.K. A prospective study of mucormycosis in north India: Experience from a tertiary care hospital. Med. Mycol. 2015, 53, 248-257. [CrossRef]

83. Chowdhary, A.; Kathuria, S.; Singh, P.K.; Sharma, B.; Dolatabadi, S.; Hagen, F.; Meis, J.F. Molecular characterization and in vitro antifungal susceptibility of 80 clinical isolates of mucormycetes in Delhi, India. Mycoses 2014, 57, 97-107. [CrossRef]

84. Prakash, H.; Ghosh, A.K.; Rudramurthy, S.; Singh, P.; Xess, I.; Savio, J.; Pamidimukkala, U.; Jillwin, J.; Varma, S.; Das, A.; et al. A prospective multicenter study on mucormycosis in India: Epidemiology, diagnosis, and treatment. Med. Mycol. 2019, 57, 395-402. [CrossRef] 